\title{
TreeSke: A Structural-Lossless Skeleton Extraction Method for Point Cloud Data of Canopy Woody Materials
}

\section{Bingxiao Wu}

Nanjing University

Guang Zheng ( $\sim$ zhengguang@nju.edu.cn )

Nanjing University

\section{Yang Chen}

State Key Laboratory of Soil and Sustainable Agriculture

Dongsheng Yu

State Key Laboratory of Soil and Sustainable Agriculture

\section{Research}

Keywords: point cloud skeleton contraction, Terrestrial Laser Scanner (TLS), canopy phenotypic characteristics, L1-median, Laplacian contraction

Posted Date: September 22nd, 2020

DOI: https://doi.org/10.21203/rs.3.rs-78239/v1

License: (9) This work is licensed under a Creative Commons Attribution 4.0 International License. Read Full License 


\section{Abstract}

\title{
TreeSke: a structural-lossless skeleton extraction method for point cloud data of canopy woody materials
}

\author{
Bingxiao Wu ${ }^{1,2}$, Guang Zheng ${ }^{2,{ }^{1 *}}$, Yang $\mathrm{Chen}^{1,3}$, and Dongsheng $\mathrm{Yu}^{1}$
}

Background: Skeletons extracted from point clouds of woody materials present canopy structural features (e.g., the inclination angle of branches) for simulating canopy interception and understory solar radiation distribution. However, existing methods cannot easily capture structure-lossless skeletons of woody organs from tree pointcloud data. To fulfill this goal, we proposed a distance-weighted method, named the TreeSke method, to iteratively contract the point cloud of canopy woody materials to their median-axis skeleton. After heuristically searching the local point set, we pointwise-extracted the tightened weights to obtain the coarse skeleton. Then, we thinned the skeleton of woody organs and optimized it by a noise filtering and recentering process.

Results: The proposed method was verified on six simulated tree models that have reference skeletons and two field-collected datasets at the plot level. The results show that the TreeSke-extracted skeletons were with higher location accuracy than using the other two tested methods. The mean offset distance, the RMSE of the offset distance, and the Hausdorff distance between the extracted and reference skeletons were less than $0.04 \mathrm{~m}$, less than $0.06 \mathrm{~m}$, and less than $0.11 \mathrm{~m}$, respectively. 
Conclusions: The extracted skeletons by the TreeSke method could nearly integrally depict the structural features of woody materials. The proposed method showed robust for noisy points and outliers, while missing data would reduce the skeleton integrity and cause deformation errors. From the extracted skeletons, users can extract the inclination-angle features of canopy woody materials, which are useful for simulating most eco-hydrological processes.

Keywords: point cloud skeleton contraction, Terrestrial Laser Scanner (TLS), canopy phenotypic characteristics, L1-median, Laplacian contraction

(1)

2

* Correspondence: zhengguang@nju. edu. cn

International Institute for Earth System Science, Nanjing University, Nanjing 210023,

\section{China}




\section{Introduction}

The structural features and distribution trends of forest canopy components, such as the canopy gap and leaf area distribution, evidently affect the canopy interception

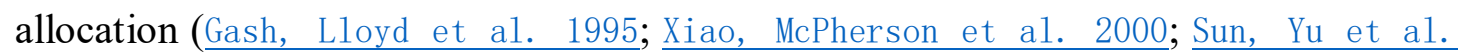
2018) and microclimate (Mitche11, Lane et a1. 2012; Maurer, Hardiman et al. 2013) in forests. Woody materials (i.e., the trunk, branches, and twigs for single tree crowns) within the forest canopy can occlude the transmission path of direct solar radiation and thus change the distribution trend of understory solar radiation (Zhu, Skidmore et al. 2018) and evaporation (Pereira, Gash et al. 2009). To model these eco-hydrological processes, previous research has widely used passive optical instruments to obtain some key canopy structural parameters, such as the effective leaf area index, gap fraction, and fractional canopy coverage (Ryu, Sonnentag et a1. 2010; Liu, Mu et a1. 2012; Hancock, Essery et a1. 2014). However, these image-based parameters cannot describe the volume and vertical distribution trend of woody materials in detail (Disney 2018; Zhu, Skidmore et al. 2018). Although using multi-spectral and multi-temporal spectral images can mask the projection area of woody materials to assess the foliage-only distribution parameters (Zou, Yan et al. 2009; Liu, Mu et al. 2012; Yan, Hu et al. 2019), they were unable to describe the distribution features of woody components along the canopy profile. More importantly, the spatial distribution, surface area, and volume of woody materials are the basis for stemflow modeling (Hanchi and Rapp 1997) and timber reserves evaluation (Bienert, Hess et al. 2014; Saarinen, Kankare et al. 2017). The inclination angle of woody organs also affects the drip water reserves, the throughfall distribution, and the canopy water storage capacity during rainfall (Xiao, McPherson et al. 2000; Schumacher and Christiansen 2015). Furthermore, the distribution of woody organs affects the wildfire risk evaluation, soil nutrient accumulation, and health status of epiphyte habitats (De Schrijver, Geudens et al. 2007; Zimmermann, Wilcke et a1. 2007).

Previous studies mainly investigated the spatial distribution of leaves, while few 
focused on describing the distribution of woody materials (Ma, Zheng et al. 2017; Liu,

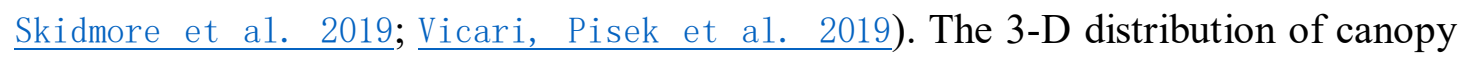
components can be precisely described as high-density discrete point cloud data using terrestrial laser scanners (TLS) (Ashcroft, Gollan et a1. 2014). Researchers have successfully classified woody and foliage points from TLS data with novel supervised and unsupervised machine learning algorithms, such as the random forest (Zhu, Skidmore et al. 2018), density-based spatial clustering of applications with noise (DBSCAN) (Ferrara, Virdis et al. 2018), and convolutional neural network (CNN)-

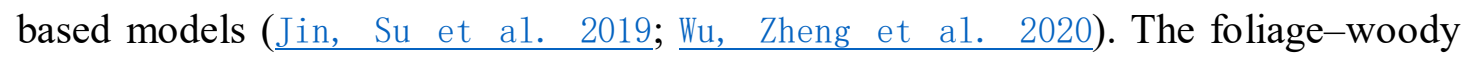
separation results can be used as a basis to accurately extract the spatial distribution features of leaves and woody materials.

To extract the geometrical features of single woody materials from discrete point cloud data, it is first necessary to segment the point cloud data (Li, Bu et al. 2017; $\mathrm{Wu}$, Wen et al. 2019). However, this step is not easy to fulfill since the point cloud data from densely overlapped branches and twigs are discrete without any topological connection information (Bremer, Rutzinger et al. 2013). Thus, in this research, we extracted the point cloud skeleton to show the structural features of woody components, such as their local inclination angles. As the definition of point cloud skeleton was still unclear, we defined it as the median-axis skeleton of the tunnel-like woody organs (Tagliasacchi, Delame et a1. 2016). Assessing the skeleton-based structural features for woody organs has the following advantages: (1) There is no need to segment single woody organs from the original discrete point cloud with complex structural features. The continuous median-axis skeleton can be beneficial to path-related analysis and length measurement; (2) Assessing the skeleton-based structural features can restrain the surface data noise caused by random tree knots, laser scattering, and missing data owing to the occlusion caused by dense leaves and overlapping branches. Some skeleton extraction methods can even repair and reconstruct the point cloud data with missing parts (Livny, Yan et al. 2010; Mei, Zhang et al. 2016); (3) using the skeleton is more beneficial for presenting the linear distribution of tunnel-like woody organs 
101 (e.g., the stems and thick branches), especially the connections between lateral branches and stems; and (4) the skeleton-based structural features are not affected by the variable local point density caused by the scanning mode (Wei, Yin et al. 2020).

Previous studies have successfully extracted skeletons from mesh-, voxel-, and graph-based point cloud models; however, only a few of these models directly ran on discrete point cloud data (Mei, Zhang et al. 2016; Tagliasacchi, Delame et al. 2016). Contracting the skeleton by a voxel-based method requires the discrete point cloud data to be discretized into numbers of voxels with a fixed spatial resolution. Then, the boundary voxels are detected to iteratively perform skeleton contraction (Song, Pang et a1. 2016). However, voxelization is unsuitable for targets with complex structural features since it would inevitably cause structural simplification and thus obtain an incomplete skeleton (Au, Tai et a1. 2008). Moreover, the extracted skeleton would be unstable for data with low point density and missing parts, even for the watertight model with holes and a bumpy surface (0zbay, Cinar et a1. 2018). The majority of skeleton extraction methods are based on the surface curvature or surface normal vector (SNV) to contract all vertices in mesh models (Au, Tai et al. 2008; Cao, Tagliasacchi et al. 2010; Tagliasacchi, Alhashim et al. 2012). Extending the mean curvature flow to extract a skeleton, the Laplacian-related contraction method directly performs on the Delaunay triangulation or Voronoi diagram for local point sets and determines the contraction and attraction weights (Cao, Tagliasacchi et al. 2010; Tagliasacchi, Delame et al. 2016; Li, Su et a1. 2020). During the iterative analysis, multiple edgecollapses are performed to remove the collapsed faces from the degenerated mesh and use surgery operation to maintain the skeleton connectivity (Qin, Han et al. 2019). The extracted skeleton can retain the local structural features of curves and holes. Although empirically setting the searching radius may reduce the skeleton extraction accuracy, the Laplacian contraction-related method shows robustness to noise and sample distribution. However, the skeleton extracted from the conjoined parts of woody organs might be outside the organs' surface (Au, Tai et al. 2008). This issue also appears in the results of some graph-based methods. After building the Reeb 

Lindenbergh et a1. 2010) graph for the discrete point cloud, running the graph-based methods can obtain the 1-D curve skeleton, which is beneficial to perform path-related analysis. The extracted results are always optimized by pruning the redundant branches and by Laplacian smoothing. The main advantage of graph-based models is that they are robust for data with low numbers of missing parts. Based on the prior knowledge about point distributions, users can repair the missing data using topological skeletons. Unfortunately, it is an ill-posed method to build a graph and mesh for woody point cloud data shown as overlapping and interlacing structural features (Song, Pang et al. 2016). For example, the mesh may contain an unreal network among points from overlapping twigs. Therefore, it is difficult to obtain the structure-lossless skeletons for canopy woody materials by using the above methods.

Point-based skeleton extraction methods contract the point cloud lacking topological information based on the local distance-related weights. Selecting a local point set is key to extracting the distribution features and determining the contraction weights for target points. The representative algorithm is the L1-median method (Huang, Wu et a1. 2013), which can extract skeletons from discrete point cloud data. Without calculating the pointwise SNVs, the L1-median method calculates the directionality degree to detect the distribution of local points and designs the regularization function to control the skeleton repulsion forces. Then, this method selects the bridge points to maintain the skeleton connectivity and re-centers the skeleton. The improved L1median methods integrate the distance field (Song, Pang et al. 2016), the L1-minimum spanning tree (MST) algorithm (Mei, Zhang et al. 2016), and the mass-driven method (Qin, Han et al. 2019) to optimize the skeleton location and the local point set selection. Most skeleton extraction methods were tested on watertight models, but rarely on the discrete point cloud of canopy woody materials with interlacing distribution features. The skeleton extraction requirements in this study are special to the above methods in the following aspects: (1) Obtaining the structural lossless skeleton of woody organs is necessary. It is the basis for assessing canopy phenotypic 
characteristics ; (2) The points from twigs are almost part of the skeleton (Digumarti, Nieto et a1. 2018), while the data from thick branches and trunks need to be iteratively thinned. Moreover, the extracted skeleton should maintain connectivity, especially at the tree forks; (3) smoothing is not suitable for skeleton extraction from branches; (4) The global setting parameters are not suitable to contract the tree point cloud data. Otherwise, adjacent twigs may be over-contracted as a pseudo branch when setting the same parameters to process data from trunks; and (5) The extraction method must show robustness to missing data, noise, outliers, and variable local point density ( $\mathrm{Li}, \mathrm{Bu}$ et a1. 2017), which are inevitable in in-situ collected forest point cloud datasets. These requirements reflect the necessity to design a specific skeleton extraction method. Thus, in the present research, we aimed to explore a new method for extracting skeletons from point cloud data of woody materials.

\section{Methods}

Based on the above requirements, the extracted skeleton should be located along the median-axis of tunnel-like stems and thick branches without noise and deformation. Moreover, the points from slim branches and twigs should be maintained as part of the skeleton. The extracted skeletons should maintain connectivity among lateral branches and stems for a single tree and without being oversmoothed. To extract the structural features of woody materials, such as the surface inclination angle, we designed a new median-axis skeleton extraction method, named the TreeSke method. This method refers to the local point distribution in the original point cloud data to obtain the pointbased skeleton for woody organs. Figure 1 shows the method's flow chart and two running samples. The following four sections will introduce this method in detail. 

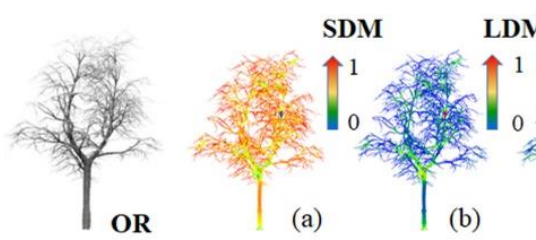

(a)
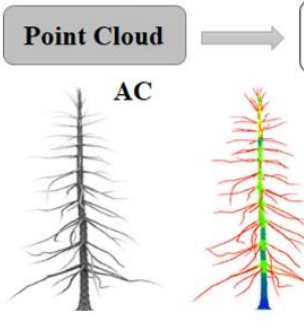

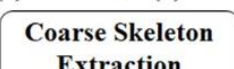
SDM $*$ LDM $\hat{1}^{1} \stackrel{y}{*} 1$ 10

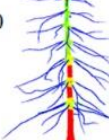

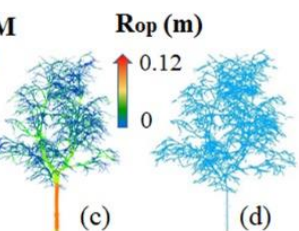
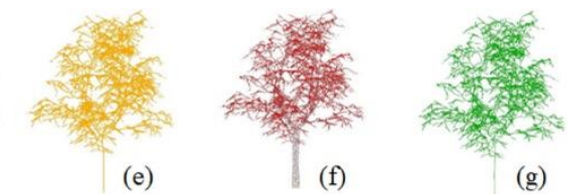

(g)

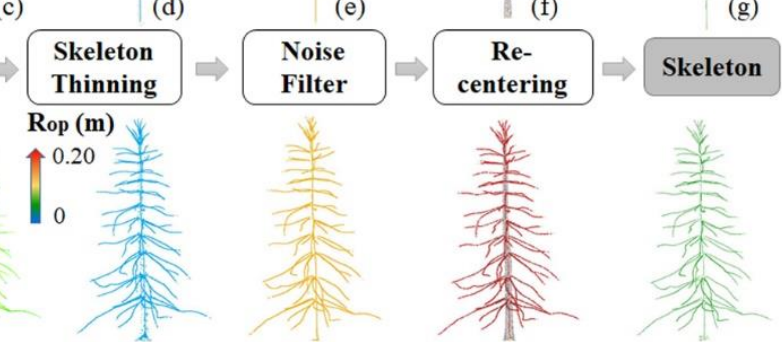

Figure 1 The flowchart of the TreeSke method for skeleton extraction and the stepby-step extraction results of two tree point cloud (OR and $\mathrm{AC}$ ) data. $\mathrm{R}_{\mathrm{op}}$ means the pointwise searching radius of local point sets in the whole point cloud data. The LDM and SDM show the linear and spherical degree measures of local point set distribution, respectively. The subfigures (a) to (c) show the evaluation results of pointwise $R_{o p}$, LDM, and SDM for two tested point cloud data.

\section{Coarse skeleton extraction}

We iteratively contracted the coarse skeleton from the original discrete point cloud after building the $\mathrm{K}$ dimensional tree (KDtree) searching index based on the pointwise 3-D coordinates. During the $\mathrm{m}_{\mathrm{th}}(\mathrm{m}=1,2, \ldots, \mathrm{M})$ iteration, we used the spherical searching method with the $\mathrm{R}_{\mathrm{a}}-\mathrm{m}$ radius to extract the pointwise local point set $P_{v e}=\left\{p_{i}\left(x_{i}, y_{i}, z_{i}\right), i=1,2, \ldots, n\right\}$. The adaptive radius $\mathrm{R}_{\mathrm{a}}$ was calculated as:

$$
R_{a}^{m}=\sqrt{d_{p}}+R_{o p}^{m-1}
$$

where $d_{p}$ is the Euclidean distance between the target point and its nearest point. The pointwise searching radius $\mathrm{R}_{\mathrm{op}}$ was set as half of the diagonal length of the $\mathrm{P}_{\mathrm{ve}}$ bounding box. During the first iteration, $R_{o p}^{0}$ was set as $0 \mathrm{~m}$. It should be noted that $\mathrm{R}_{\mathrm{op}}$ is related to $\mathrm{R}_{\mathrm{a}}$, but they are different metrics. As shown in Figure 1, points from trunks had larger values of $\mathrm{R}_{\mathrm{op}}$ than those from branches and twigs after the $\mathrm{m}_{\text {th }}$ iteration, which avoided to search nearby points from another woody organ for the target points. Thus, setting pointwise $R_{o p}$ is essential to maintain the local structural features of twigs and slim branches during the coarse skeleton extraction. 
For a target point, we calculated two geometrical features, the linear degree measure (LDM) (Huang, Wu et a1. 2013; Mei, Zhang et al. 2016) and the spherical degree measure (SDM), to represent the distribution pattern of its $\mathrm{P}_{\mathrm{ve}}$ by the covariance matrix $\left(\mathrm{C}_{\text {cov }}\right)$ of $n(n \geqslant 3)$ coordinates:

$$
\begin{gathered}
\mathrm{C}_{\mathrm{cov}}=\frac{1}{n}\left({ }_{p}^{\mathrm{V}}-\stackrel{\mathrm{v}}{p}_{i}\right)^{T}\left(\stackrel{\mathrm{V}}{p}_{i}-\stackrel{\mathrm{v}}{\mathrm{v}}_{i}\right), \\
\mathrm{\mathbf {v }} \\
\mathrm{p}=\frac{1}{n} \sum_{i=1}^{n}\left(x_{i}, y_{i}, z_{i}\right)^{\mathrm{T}},
\end{gathered}
$$

where T means the transposed matrix; and $\lambda_{0}, \lambda_{1}$, and $\lambda_{2}$ are ordered eigenvalues of $\mathrm{C}_{\text {cov. }}$ Then,

$$
L D M=\frac{\lambda_{2}}{\lambda_{0}+\lambda_{1}+\lambda_{2}}\left(\lambda_{2} \geq \lambda_{1} \geq \lambda_{0}\right)
$$

If a $\mathrm{P}_{\mathrm{ve}}$ has fewer than three points, we set $\mathrm{LDM}=1$ and $\mathrm{SDM}=0$ as the attributes for the target point. For points that are on linear-distributed woody organs, their LDMs are near 1, and their SDMs are near 0. On the contrary, for points from a local planerdistributed surface, such as trunks and thick branches, the related LDMs are near 0 and the related SDMs are near 1. The pointwise LDM and SDM further formed a weight to optimize the $\mathrm{R}_{\mathrm{op}}$ of each point and thus avoided overcontraction for the point cloud at the intersection parts of woody organs and maintained the original distribution of points from twigs and slim branches:

After the initial iteration, each point had two geometrical attributes. During the following iterations, these two features were point-by-point recalculated according to the local point distribution of $\mathrm{P}_{\mathrm{ve}}$ extracted from the contracted skeleton. If point $\mathrm{O}$ has a larger LDM in the $\mathrm{m}_{\mathrm{th}}$ iteration than in the $(\mathrm{m}-1) \mathrm{th}(\mathrm{m}>1)$ iteration, its coordinate $\mathrm{P}_{\mathrm{o}}$ was relocated by the weighted median-based method as:

$$
W=\left(1+e^{(L D M-S D M) /(L D M+S D M)}\right)^{-1}
$$


Through several tests, setting $M=3$ was shown to effectively extract the coarse skeletons. During this step, the coarse skeletons and pointwise LDMs and SDMs were heuristically extracted from point cloud data without setting any empirical parameters.

\section{Thinning the coarse skeleton}

Through the iterative contraction, we obtained the coarse skeleton and determined the pointwise $\mathrm{R}_{\mathrm{op}}$. However, the skeleton points from the tunnel-like trunk and lateral branches (inset (d) of Figure 1) still needed to be further thinned. Since the coarse skeleton is wrapped by the original point cloud, the shortest pointwise Euclidean distance between the coarse skeleton and the original data $\left(\mathrm{D}_{\mathrm{co}}\right)$ was set as the contraction radius during the thinning process.

(1) We built the Kdtree index for the original point cloud data $\left(\mathrm{KD}_{\mathrm{o}}\right)$ and the coarse skeleton $\left(\mathrm{KD}_{\mathrm{c}}\right)$, respectively. The $\mathrm{KD}_{\mathrm{o}}$ and $\mathrm{KD}_{\mathrm{c}}$ were used to calculate the pointwise $\mathrm{D}_{\mathrm{co}}$ for points in a coarse skeleton. In the following steps, if a point had a $\mathrm{D}_{\mathrm{co}}$ larger

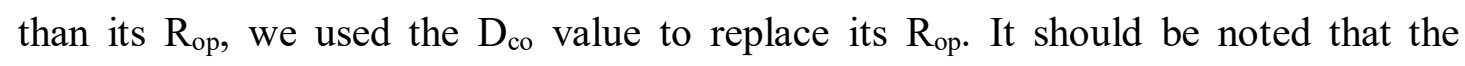
pointwise $R_{o p}$ (or $D_{c o}$ ) was rounded up to 2 decimal places to increase the running efficiency.

(2) In the $V_{\text {th }}$ iteration $\left(V \leqslant 3\right.$ in this study), we spherically searched the $P_{v e}$ for each point in the coarse skeleton with a radius of $R_{o p}$ (or $D_{c o}$, larger than 0 ). Then, we calculated the LDM for each point according to its $\mathrm{P}_{\mathrm{ve}}$. Gradually reducing $\mathrm{R}_{\mathrm{op}}$ could effectively restrain the skeleton deformation and fracture issues for the point cloud from tree fork parts during the thinning process. Thus, we set the pointwise LDM as the weight to optimize $\mathrm{R}_{\mathrm{op}}$ as:

$$
R_{o p}=R_{o p} \cdot(1-L D M)
$$

For most skeleton points from linear-distributed twigs and slim branches with LDM near 1 , their $\mathrm{R}_{\mathrm{op}}$ converged rapidly to 0 to avoid further being moved.

(3) We used the distance-weighted average algorithm to relocate the points with $R_{o p}$ higher than 0 and thin the coarse skeleton gradually. For a target point, its weight $\left(\mathrm{W}_{\text {thin }}\right)$ 
to the mean coordinate of nearby (n-1) points in a $\mathrm{P}_{\mathrm{ve}}$ during the $\mathrm{V}_{\text {th }}$ iteration was:

$$
\begin{gathered}
W_{\text {thin }}=\frac{e_{i}^{D_{c o}}}{\sum_{i=1}^{n-1} e_{i}^{D_{c o}},} \\
P_{o}=\frac{P_{v e} \cdot W_{\text {thin }}}{n-1} .
\end{gathered}
$$

Thus, in a $\mathrm{P}_{\mathrm{ve}}$, points with larger $\mathrm{D}_{\mathrm{co}}$ would have higher $\mathrm{W}_{\text {thin }}$ to relocate the target point coordinate. For a point with $\mathrm{R}_{\mathrm{op}}$ equal to 0 , its $\mathrm{W}_{\text {thin }}$ was set to be larger than 0 to avoid deviation in the gradually thinned skeleton.

In the thinned skeleton, we found that some points from stems with low $\mathrm{R}_{\mathrm{op}}$ would became outliers. Additionally, some skeleton points from tree forks or roots were shown as lumped and redundant distribution trends. Thus, we needed to filter-out noisy points from the skeleton extraction results.

\section{Noise filtering}

We set the distance-based threshold to remove outliers and redundant points from the thinned skeleton:

(1) For point $P_{o}$ in the thinned skeleton, we searched its nearby $k$ points; here, $k$ was set as 9. After summing the Euclidean distance from $\mathrm{P}_{\mathrm{o}}$ to its $\mathrm{k}$ neighboring points $\left(\mathrm{SUM}_{\mathrm{d}}\right)$, we added the $\mathrm{SUM}_{\mathrm{d}}$ as an attribute to $\mathrm{P}_{\mathrm{o}}$. Meanwhile, we removed the points with LDM lower than 0.85 , which were always shown to be the redundant points at the tree forks.

(2) According to the pointwise $\mathrm{SUM}_{\mathrm{d}}$, we used the threshold filtering method to detect noise and outliers. After several tests, the threshold $\mathrm{TH}_{\text {SUM }}$ was set as the $99.5 \%$ quantile of the ascending ordered $\mathrm{SUM}_{\mathrm{d}}$ of all skeleton points. Each point with a SUM $\mathrm{S}_{\mathrm{d}}$ larger than $\mathrm{TH}_{\text {Sum }}$ was removed from the thinned skeleton.

(3) The above two steps were run for S iterations. During each iteration, we calculated the $\mathrm{SUM}_{\mathrm{d}}$ for each point by searching its nearby k points. After filtering out the noise and outliers with a $\mathrm{SUM}_{\mathrm{d}}$ larger than $\mathrm{TH}_{\mathrm{SUM}}$ from the skeleton points, the remaining points were linearly distributed. After several tests, we set $\mathrm{S}$ as 3 times. 
After removing noise and some redundant skeleton points, we found that part of the thinned skeleton deviated from the median axis of wood organs described in the point cloud data. Thus, it was necessary to re-center these points to optimize the skeleton extraction results of stems and thick branches and thus to be beneficial for calculating the local geometrical features of woody materials (Tagliasacchi, Alhashim et a1. 2012; Huang, Wu et al. 2013). To reduce the computation cost, we used the octree-based tool embedded in the CloudCompare software (http://cloudcompare.org/) to sub-sample the skeletons. The sub-sample class was set as 10 to maintain the local geometrical features and keep the integrity of point-based skeletons.

For point $\mathrm{H}_{\mathrm{o}}$ in the original point cloud data, we searched for its nearest point $\mathrm{H}_{\mathrm{s}}$ in its related sub-sampled skeleton point sets. The index of $\mathrm{H}_{\mathrm{s}}(\mathrm{INDEX})$ in the skeleton point set was added as an attribute to $\mathrm{H}_{\mathrm{o}}$. Then, we extracted the nearby points from the original point cloud data around $\mathrm{H}_{\mathrm{s}}$ using the pointwise INDEX. If the number of nearby points in $\mathrm{H}_{\mathrm{o}}$ for $\mathrm{H}_{\mathrm{s}}$ was higher than 4, the threshold to meet the requirement of the ellipse-fitting algorithm for discrete points (Halır and Flusser 1998), we used the leastsquares fitting method to relocate $\mathrm{H}_{\mathrm{s}}$ with the coordinates of its nearby points in $\mathrm{H}_{\mathrm{o}}$. Otherwise, we kept the coordinate of $\mathrm{H}_{\mathrm{s}}$ in the skeleton. Finally, we obtained the skeleton from the original point cloud data. As woody materials often show interlacing distribution within the upper tree crowns, it is infeasible to further construct the topological graphs for the extracted point-based skeletons.

\section{The results evaluation}

To evaluate the skeleton extraction accuracy, most studies used qualitative (visually) assessment and quantitative evaluation metrics, such as skeleton integrity, centeredness, re-constructability, and the offset distance (OFD) between the extracted and reference skeleton of scan targets. Here, we combined two types of assessments to evaluate our skeleton extraction results and those extracted by the other two methods: the L1-median (Huang, Wu et a1. 2013) and Laplacian contraction (Cao, Tagliasacchi 
et al. 2010) methods. Each extracted skeleton was embedded into the original point cloud data to show its integrity and connectivity among different woody organs. In this study, we extracted the reference topological skeletons of all simulated mesh-based tree models. The extraction method is briefly introduced in Section 4. Therefore, we calculated the OFD between each skeleton point $p_{o}$ (totally $t$ points) to its nearest reference skeleton vertex p', and other statistical metrics, including the mean and maximum OFD, and the RMSE of pointwise OFD values (OFD $\mathrm{RMSE}_{\text {): }}$

$$
O F D_{R M S E}=\sqrt{\frac{1}{t} \sum_{n=1}^{t}\left(p_{0}-p^{\prime}\right)\left(p_{0}-p^{\prime}\right)^{\mathrm{T}}} .
$$

We also calculated the Hausdorff distance (HDF) between the extracted (E) and reference (S) skeletons for each simulated tree model (Taha and Hanbury 2015; Tagliasacchi, Delame et a1. 2016; Lin, Liu et al. 2020).

$$
\begin{gathered}
H D F=\max \{h(E, S), h(S, E)\}, \\
h(E, S)=\max \left(p_{o} \in E\right) \min \left(p^{\prime} \in S\right)\left\|p_{o}-p^{\prime}\right\|, \\
h(S, E)=\max \left(p^{\prime} \in S\right) \min \left(p_{o} \in E\right)\left\|p^{\prime}-p_{o}\right\| .
\end{gathered}
$$

Furthermore, we assessed the inclination angle (IA)s for each skeleton point:

$$
I A=\arctan \left(\frac{z_{\text {range }}}{\sqrt{x_{\text {range }}^{2}+y_{\text {range }}^{2}}}\right) .
$$

Here, $x_{\text {range, }} y_{\text {range, }}$ and $z_{\text {range }}$ are the side length (x-axis, $y$-axis, and $z$-axis) of the bounding box contained the target point and its nearest two skeleton points, respectively. Then, we point-by-point broadcasted the IA to the original point cloud data according to the nearest neighbor rule. We also evaluated the SNV for each point in the original point cloud according to the coordinates set of its local nearest 100 points. The pointwise SNV was also used to calculate the local inclination angle.

For each point in the original point cloud data, we assessed the absolute difference between its skeleton- and SNV-based inclination angle (Li, Su et al. 2018; Vicari, Pisek et a1. 2019). As the field-collected datasets did not have reference skeletons, the comparison between the skeleton- and SNV-based inclination angle was only suitable 
for the simulated tree point cloud data. The TreeSke and skeleton location accuracy evaluation methods were fulfilled by the SciPy v1.5.1 package (https://scipy.org/) in Python 3.5 program environment, except the down-sampling process described in Section 3.4 .

\section{The validation datasets}

To verify the TreeSke method, we prepared two types of datasets: the in-situ collected tree point cloud data at plot level and simulated data converted from the meshbased single tree models. The Leica HDS 3000 TLS (Leica Geosystems AG, Heerbrugg, Switzerland) was used to collect the point cloud data from two plots, including one homogeneous grown with the Masson's Pines (Pinus massoniana, MP) and another with Chinese aspens (Populus adenopoda, CA). The MP plot is located in the red soil ecological experimental station of the Institute of Soil Science, Chinese Academy of Sciences $\left(28^{\circ} 12^{\prime} 21^{\prime \prime} \mathrm{N}, 116^{\circ} 55^{\prime} 42^{\prime \prime} \mathrm{E}\right)$ in Jiangxi province, China. The CA plot is set in the Baima research and experimental forest site $\left(31^{\circ} 36^{\prime} 51^{\prime \prime} \mathrm{N}, 119^{\circ} 11^{\prime} 8^{\prime \prime} \mathrm{E}\right)$ in Jiangsu province, China.

During the data collection, we set the predefined angular resolution as $0.005 \mathrm{~m} @$ $10 \mathrm{~m}$. Five comprehensive field-of-view (i.e., horizontal: $0^{\circ}$ to $360^{\circ}$; vertical: $-45^{\circ}$ to $90^{\circ}$ ) laser scan were obtained in each plot contained one center and four corner scan locations. After data registration by the Leica Cyclone V9.0 software (Leica Geosystems AG Heerbrugg, Switzerland), we used the Cloudcompare software to filter the ground points out (Zhang, Qi et al. 2016). In the next step, we subsampled the original data with the $0.01-\mathrm{m}$ distance to filter redundant points out. To extract the woody points, we used the FWCNN model (Wu, Zheng et al. 2020) to classify the original tree data and removed the foliage points. Furthermore, we visually detected and removed the misclassified points.

As the field-collected point cloud data did not contain the reference skeletons, we only qualitatively evaluated the skeleton integrity for the extracted results of CA and 
MP datasets. To quantitatively assess the median-axis skeleton location accuracy, we resampled six mesh-based tree models (stored as .obj format) provided in the SpeedTree software (https://store.speedtree.com/cinema/) to discrete point cloud datasets by the Cloudcompare software. They include four broadleaved and two coniferous tree models with different structural features of woody organs (Figure 2). After removing the foliage parts in each tree model, we converted the woody parts into point cloud data (Digumarti, Nieto et a1. 2018). To reduce the computation cost during the skeleton extraction, each resampled point cloud data has 250,000 points. Thus, their point density was different. Then, we used the extraction tool embedded in the Starlab software (https://github.com/OpenGP/starlab) to obtain the topological reference skeletons from mesh-based tree models and maintain vertexes in each reference skeleton. The related statistical information of each point cloud data is shown in Table 1.

Owing to the noise and missing parts inevitably exist in the in-situ collected data, we randomly added the Gaussian noise and outliers to the point cloud converted from the simulated tree models (Digumarti, Nieto et al. 2018). Moreover, we randomly removed parts of point cloud data (Mei, Zhang et al. 2016). These low-quality datasets (Figure 5) were used to verify the robustness of the TreeSke method.

Table 1 The statistical information of all tested point cloud datasets. For each mesh-based tree model, we measured its crown diameter. The plot size shows the side length of the CA and MP datasets at the plot level. The point density means the average number of points in a sphere zone with the $0.05-\mathrm{m}$ radius. In the Type column, $\mathrm{B}$ and $\mathrm{C}$ mean the coniferous and broadleaved trees, respectively.

\begin{tabular}{|c|c|c|c|c|c|}
\hline Data & $\begin{array}{c}\text { Number of trees } \\
(\#)\end{array}$ & $\begin{array}{l}\text { Height } \\
\text { (m) }\end{array}$ & $\begin{array}{l}\text { Crown diameter / Plot } \\
\text { size } \\
\text { (m) }\end{array}$ & $\begin{array}{c}\text { Point } \\
\text { density } \\
\text { (pts/unit) }\end{array}$ & Type \\
\hline $\mathrm{AC}$ & 1 & 6.81 & 3. 91 & 26 & C \\
\hline GA & 1 & 6.41 & 5.20 & 10 & B \\
\hline SW & 1 & 6.57 & 3.28 & 15 & B \\
\hline OR & 1 & 2. 37 & 1.96 & 75 & B \\
\hline HS & 1 & 4. 12 & 2.63 & 49 & C \\
\hline
\end{tabular}




\begin{tabular}{cccccc}
\hline LP & 1 & 7.39 & 5.18 & 17 & B \\
MP & 54 & 10.62 & $10 \times 10$ & 16 & C \\
CA & 15 & 11.34 & $11 \times 11$ & 16 & B \\
\hline
\end{tabular}

387

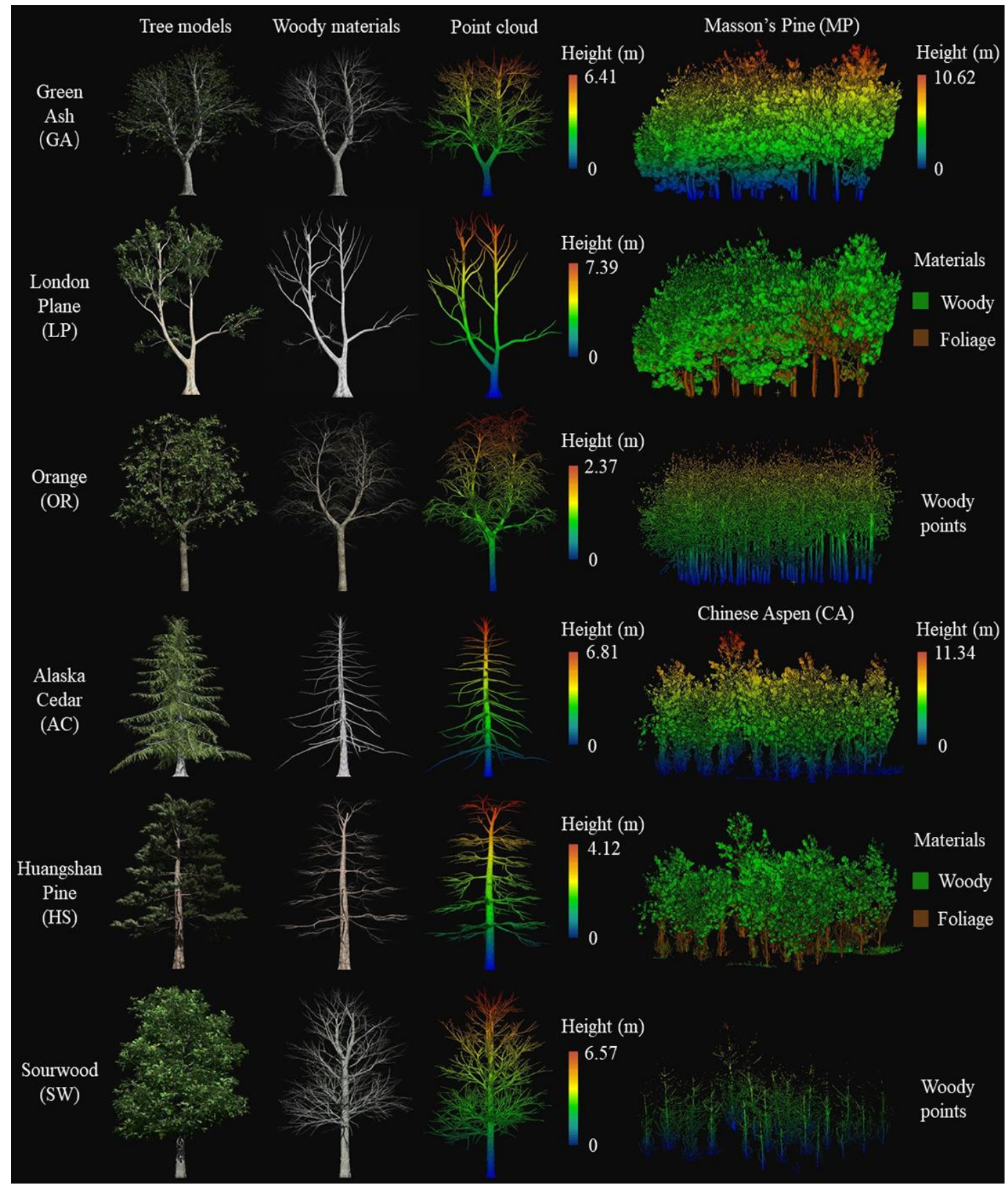

389 Figure 2 The point cloud of woody materials converted from six simulated single tree 390 mesh models and those collected from MP and CA plots. 


\section{Results}

\section{Visual evaluation}

We embedded the extracted skeletons into the original point cloud data to qualitatively evaluate their integrity and connectivity. As shown in the insets of Figure 3 , the extracted skeletons can integrally present the structural features of interlacing branches and twigs in the simulated tree models. The skeleton of single woody organs maintained good continuity, while some outliers and breaking points existed at some intersection parts of woody materials with large curvature. Additionally, some coneshaped skeleton point clusters occurred at the root where the radius of woody materials changed gradually. We thought that the local skeleton points of roots were reasonably shown to have a non-vertical lumpy distribution since their SNVs were not parallel to the ground. However, the TreeSke method could not extract the skeleton from missing parts which lacked the reference points for calculating the contraction weights. The skeleton points of overlapping twigs may be distorted when setting an overlarge radius to extract their local point sets. As the pointwise searching radius was heuristically set according to Section 3.1, it is infeasible to subjectively adjust them.

We also performed a test by extracting the plot-level skeleton of woody materials for field-collected point cloud data. As shown in the insets (m) to (p) of Figure 3, although the lateral branches of neighboring trees in two plots were interlacing, the extracted skeletons presented the structural features of those describable branches and stems. Due to the occlusion effects caused by dense and overlapping leaves and woody organs within the forest canopy, the point cloud might not show all woody organs in the upper tree crowns. For those missing parts, such as the points of the treetop in the MP plot and the incomplete twigs in the CA plot, using the TreeSke method cannot extract their skeletons. 
(a) $\mathrm{AC}$

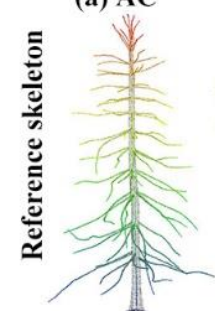

(g)

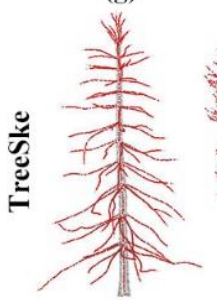

(h)

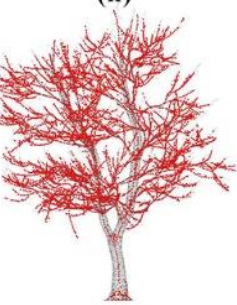

(m) MP plot

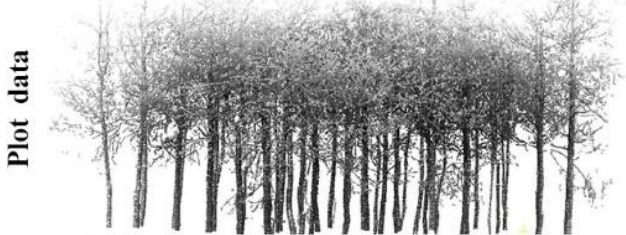

(o)

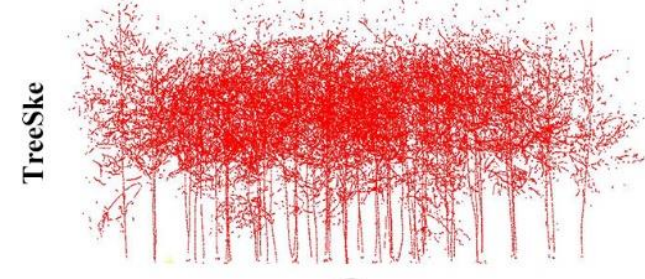

(d) $\mathrm{OR}$

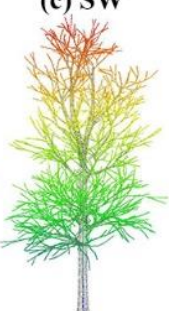

(i)
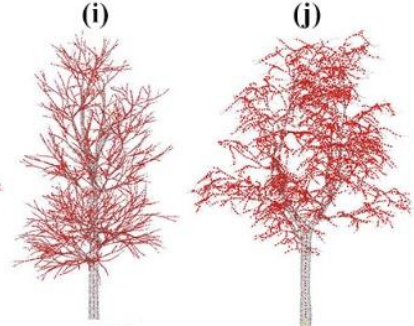

(j)

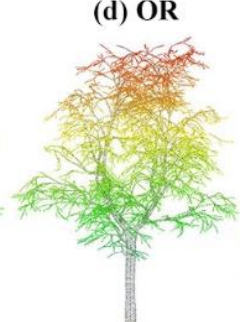

(1)

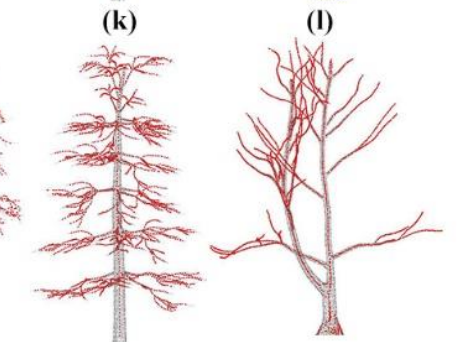

(n) CA plot

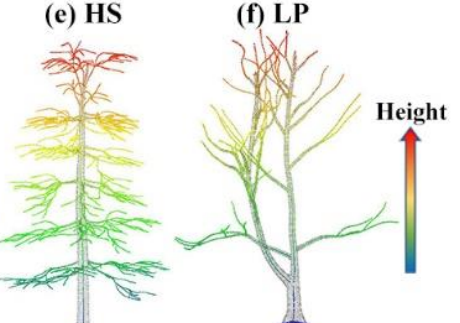

(k)

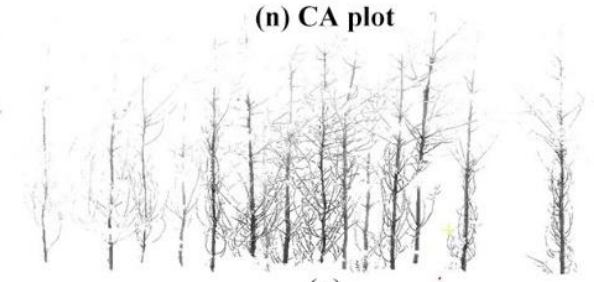

(p)

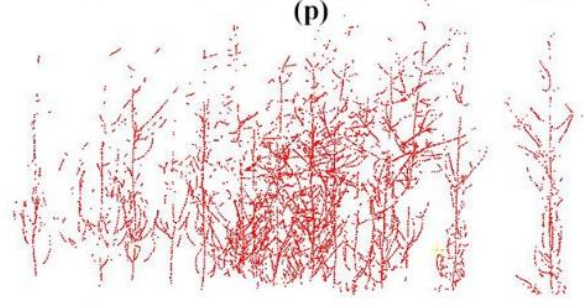

skeleton points

Figure 3 The TreeSke-extracted results of simulated (single tree level) and fieldcollected (plot-level) tree point cloud datasets.

\section{The skeleton extraction accuracy}

According to the reference skeletons, we pointwise calculated the OFD from each extracted skeleton to its corresponding reference skeleton. The pointwise OFDs and the related statistical information, including the mean OFD, the RMSE of OFD (OFD RMSE$_{\text {, }}$, and the HDF between the reference and extracted skeleton point sets, are shown in Figure 4. Using the TreeSke method could extract the approximate structural-lossless skeletons from linear-distributed lateral branches and twigs. The OFD $\mathrm{RMSES}_{\mathrm{S}}$ of extracted skeletons were lower than $0.06 \mathrm{~m}$. The largest OFD values were about $0.18 \mathrm{~m}$ for the extracted skeleton of the LP and GA models. Moreover, the mean OFD between the extracted and reference skeletons were lower than $0.04 \mathrm{~m}$. The HDFs between the 
reference and extracted skeletons were lower than $0.11 \mathrm{~m}$. We found most skeleton deviations occurred at the frustum-like tree roots and some conjunction parts of different woody organs. The results present that the TreeSke-extracted skeletons could describe the complex structural features of interlacing branches, while some tiny twigs shown in the OR and GA point cloud data may be misrecognized as outliers and removed. We found the reference skeletons extracted by the Starlab software shown some excessive extension at the tip of twigs, within which some junction parts between branches and stems were fractured. Therefore, it was infeasible to use the reference skeletons to evaluate the integrity of our extracted results.

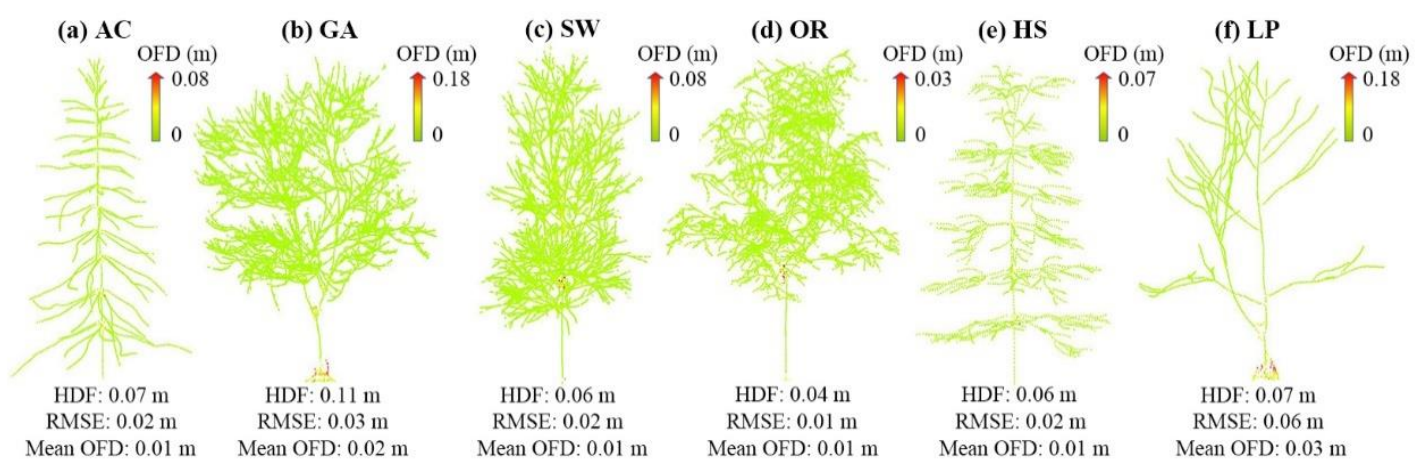

Figure 4 The pointwise OFD of TreeSke-extracted skeleton points. In each inset, the gradient colors show the variation trend of pointwise OFD values, which means the offset distance from each extracted skeleton to its corresponding reference skeleton.

For the simulated point cloud that with the Gaussian noise, outliers, and missing parts, we found their point-to-point skeleton extraction accuracy was decreasing according to the higher mean OFD, OFD RMSE, and HDF between the extracted and referenced skeletons. As shown in Figure 5 and Table 2, the extracted accuracy dropped more severely for the skeletons of AC and HS point cloud with the HDF increased 0.03 $\mathrm{m}$ and $0.05 \mathrm{~m}$, respectively, and the largest OFD increased by $0.13 \mathrm{~m}$. While the related statistical metrics were nearly unvaried for the GA and OR datasets. The skeleton points with larger OFD values were always at the missing parts from stems and roots, where the extracted skeletons presented obvious deformation. At these missing parts, the extracted skeleton points showed lumpy or looped distribution. Therefore, we inferred that the TreeSke method was sensitive to the missing data from trunks, but it kept robust to the added noisy points and outliers. Even if parts of point cloud data from lateral 

these missing parts.

(a) $\mathrm{AC}$
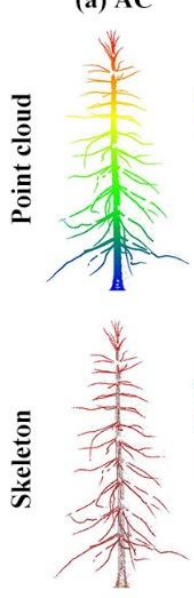

(b) GA
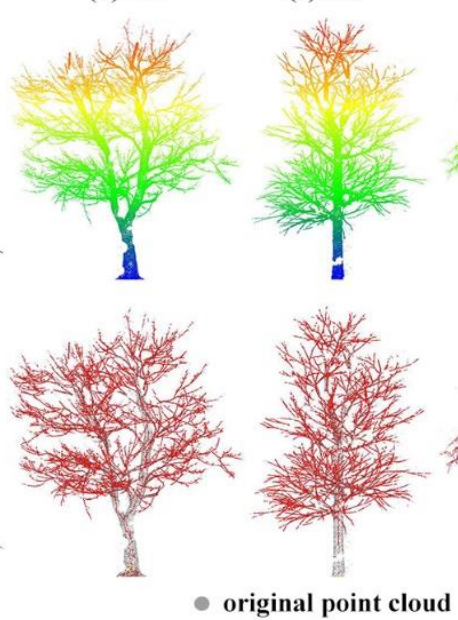

(c) $\mathrm{SW}$

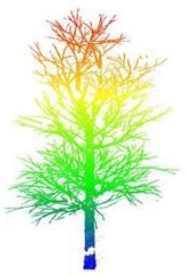

(d) OR
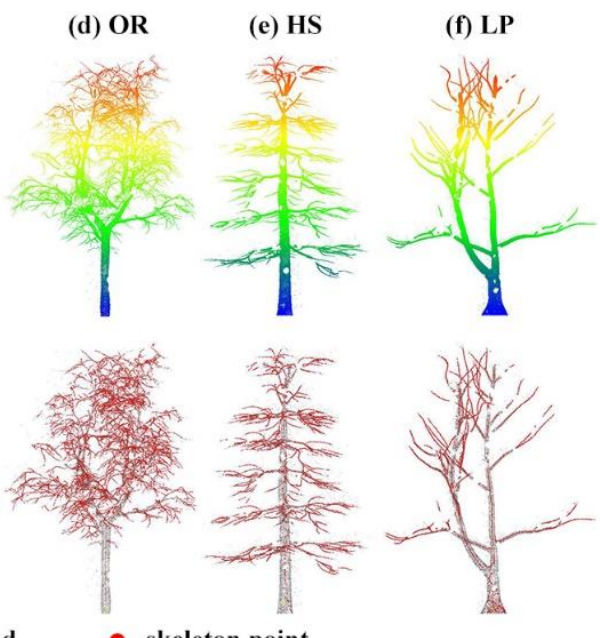

458

459

460

461

462

463

464

465
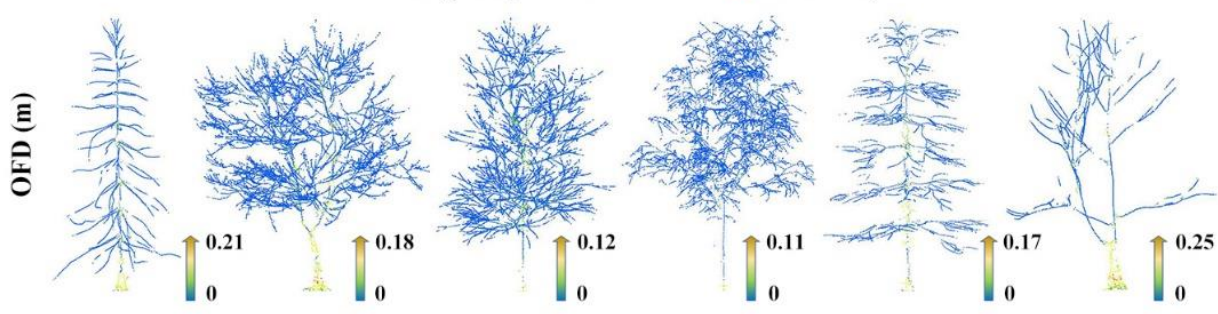

Figure 5 The TreeSke-extracted skeletons and pointwise OFD values of point cloud data with noisy points, outliers, and missing parts.

Table 2 The location accuracy comparison between those skeletons extracted from the point cloud data with noise and free to noise. In this table, HDF means the Hausdorff distance between the extracted and reference skeletons from each tree model. $0 \mathrm{FD}_{\text {RusE }}$ means the root mean square error among the point-to-point OFD values.

\begin{tabular}{ccccccc}
\hline Data & \multicolumn{3}{c}{ Free to noi se } & \multicolumn{3}{c}{ With noise } \\
\cline { 2 - 7 } & $\begin{array}{c}\text { OFD } \\
(\mathrm{m})\end{array}$ & $\begin{array}{c}\text { Mean 0FD } \\
(\mathrm{m})\end{array}$ & $\begin{array}{c}\mathrm{HDF} \\
(\mathrm{m})\end{array}$ & $\begin{array}{c}\text { OFD } \\
(\mathrm{m})\end{array}$ & $\begin{array}{c}\text { Mean } 0 \mathrm{FD} \\
(\mathrm{m})\end{array}$ & $\begin{array}{c}\text { HDF } \\
(\mathrm{m})\end{array}$ \\
\hline $\mathrm{AC}$ & 0.02 & 0.01 & 0.07 & 0.06 & 0.02 & 0.10 \\
$\mathrm{GA}$ & 0.03 & 0.02 & 0.11 & 0.04 & 0.02 & 0.11 \\
$\mathrm{SW}$ & 0.02 & 0.01 & 0.06 & 0.02 & 0.01 & 0.08 \\
OR & 0.01 & 0.01 & 0.04 & 0.01 & 0.01 & 0.04 \\
$\mathrm{HS}$ & 0.02 & 0.01 & 0.06 & 0.04 & 0.02 & 0.11 \\
$\mathrm{LP}$ & 0.06 & 0.03 & 0.07 & 0.12 & 0.05 & 0.08 \\
\hline
\end{tabular}




\section{The pointwise inclination angle}

We calculated the absolute difference between the pointwise inclination angle calculated by the SNV of woody organs (SNV-based) and the TreeSke-extracted skeleton points (skeleton-based). The results show that the pointwise absolute inclination angle differences by using two methods are evident (Table 3). The mean differences are lower than $30^{\circ}$. Over $31 \%$ of the number of points is with the angle difference values lower than $15^{\circ}$. However, for the AC and HS data, we found there were over $28 \%$ of the number of points with the angle difference over $45^{\circ}$. In addition, the angle differences were higher for those points from trunks.

Through visual assessment, we found these two methods had their own advantages and disadvantages in evaluating the surface inclination angle of wood organs. As shown in Figure 6, the SNV-based method could not correctly assess the pointwise inclination angles from twigs, those woody organs growing parallel to the ground, and the tree forks. Moreover, it was irrational that nearby points from the same branch were always with different inclination angles. We inferred that the extracted local point sets for these points were from their surrounding woody organs when setting an overlarge searching radius, rather than that of the branch where each point is located. While the skeletonbased inclination angles from tree forks were lower than their authentic values. When evaluating the local inclination angles of non-trunk woody materials, using the skeleton-based method could obtain more accurate results. However, it would be better to calculate the surface inclination angle of trunks and thick branches base on their local SNVs. We will discuss how to optimize the calculation methods of local surface inclination angle for woody materials. 


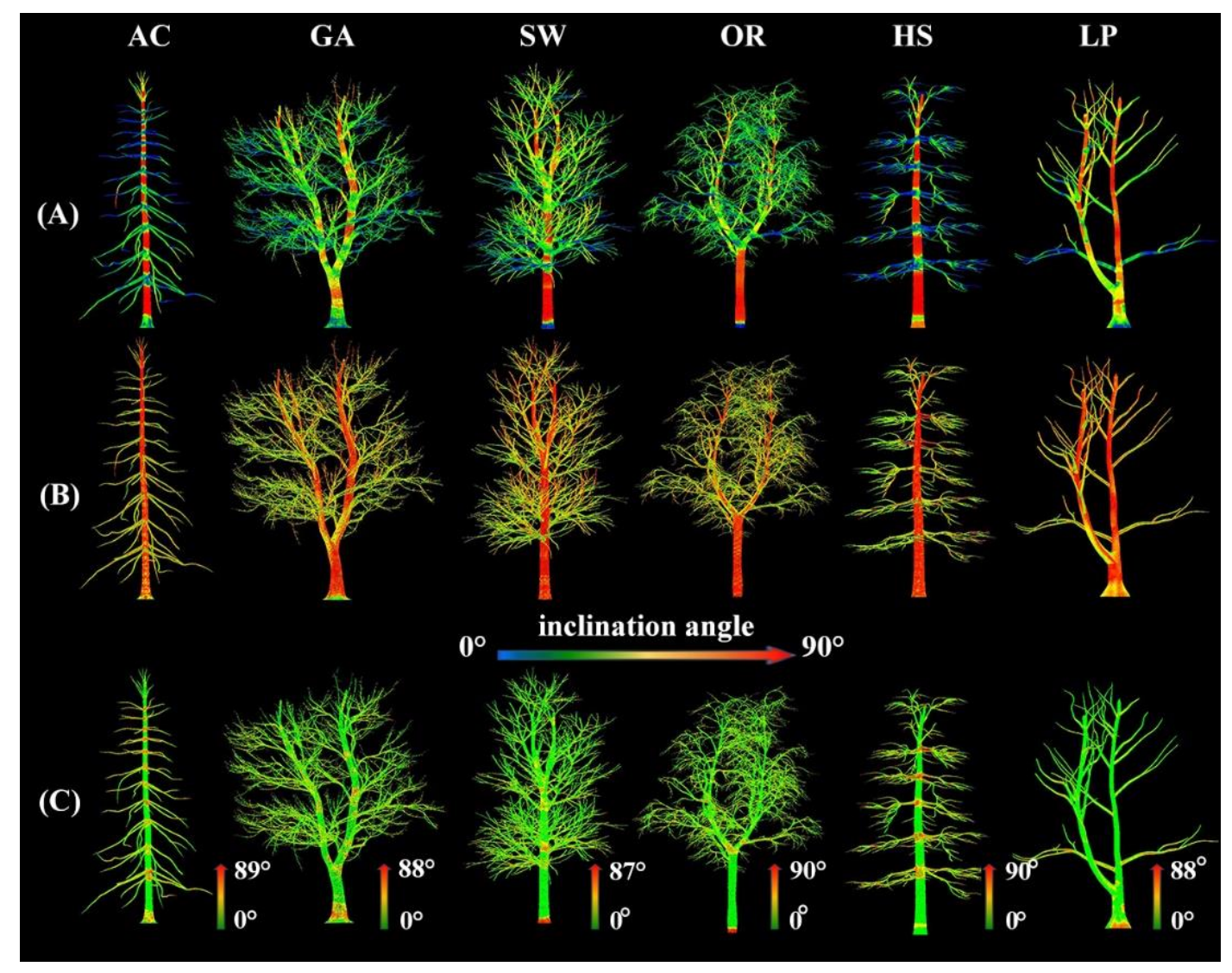

Figure 6 The insets in row (A) and (B) show the pointwise surface inclination angles 491 calculated by the skeleton- and SNV-based methods, respectively. In row (C), these 492 insets show the pointwise absolute difference of surface inclination angle evaluated 493 by two methods.

Table 3 Some statistical information about the pointwise absolute differences 497 between the SNV- and skeleton-based inclination angle. The range of absolute 498 angle difference is from $0{ }^{\circ}$ to $90^{\circ}$. We surveyed the proportion of points to the 499 whole data in every $15^{\circ}$ intervals.

\begin{tabular}{cccccccc}
\hline Data & $\begin{array}{c}\text { Mean } \\
\left({ }^{\circ}\right)\end{array}$ & $\begin{array}{c}{\left[0^{\circ}, 15\right.} \\
\left.{ }^{\circ}\right)\end{array}$ & $\begin{array}{c}{\left[15^{\circ}, 30\right.} \\
\left.{ }^{\circ}\right)\end{array}$ & $\begin{array}{c}{\left[30^{\circ}, 45\right.} \\
\left.{ }^{\circ}\right)\end{array}$ & $\begin{array}{c}{\left[45^{\circ}, 60\right.} \\
\left.{ }^{\circ}\right)\end{array}$ & $\begin{array}{c}{\left[60^{\circ}, 75\right.} \\
\left.{ }^{\circ}\right)\end{array}$ & $\begin{array}{c}{\left[75{ }^{\circ}, 90\right.} \\
\left.{ }^{\circ}\right]\end{array}$ \\
\hline AC & 29.28 & 36.63 & 18.57 & 16.77 & 15.49 & 10.10 & 2.45 \\
GA & 28.10 & 31.24 & 26.53 & 20.68 & 14.50 & 6.46 & 0.60 \\
SW & 24.98 & 38.57 & 25.39 & 18.72 & 11.50 & 4.95 & 0.86 \\
OR & 26.55 & 36.77 & 23.54 & 19.06 & 13.53 & 6.18 & 0.92 \\
HS & 29.78 & 36.77 & 16.46 & 15.96 & 14.47 & 11.83 & 4.52 \\
LP & 19.49 & 53.57 & 20.65 & 14.47 & 7.48 & 3.29 & 0.54 \\
\hline
\end{tabular}




\section{Discussions}

\section{Comparison with existing methods}

We compared the skeleton extraction accuracy of our proposed method with those of two methods, namely the L1-median (Huang, Wu et al. 2013) and Laplacian contraction (Cao, Tagliasacchi et al. 2010) methods. Considering that it is ill-posed to convert field-collected discrete point cloud data into a mesh- or graph-based tree model, some representative skeleton extraction methods, such as the ROSA algorithm (Tagliasacchi, Zhang et al. 2009), were not tested. The accuracy evaluation metrics were described in Section 3.5. We found that the skeletons extracted by the L1-median method could capture the skeleton points from cylinder-like woody organs, while the skeletons of slim branches and twigs were almost absent (Figure 7). This issue was also found in other studies (Song, Pang et al. 2016; Wu, Wen et al. 2019). Although the L1-median method precisely extracted the local median-axis skeleton points with OFD RMSE $<0.13 \mathrm{~m}$, mean OFD $<0.11 \mathrm{~m}$, and HDF $<0.17 \mathrm{~m}$ (Table 4), this method could not extract the structural lossless skeleton from interlacing woody materials, and thus could not be used to assess the surface inclination angle of lateral branches. More importantly, we found that the L1-median-based skeletons were variable and sensitive to the selection of initial sample points (Qin, Han et al. 2019). For tree point cloud data with interlacing twigs, the skeleton extraction results would be different when selecting diverse sample point sets. For a high-quality tree model without interlacing branches, such as the LP and AC models, using the L1-median method can achieve satisfactory skeleton extraction results. 
(a) $\mathrm{AC}$

(b) GA

(c) $\mathrm{SW}$

(d) OR

(e) HS

(f) LP
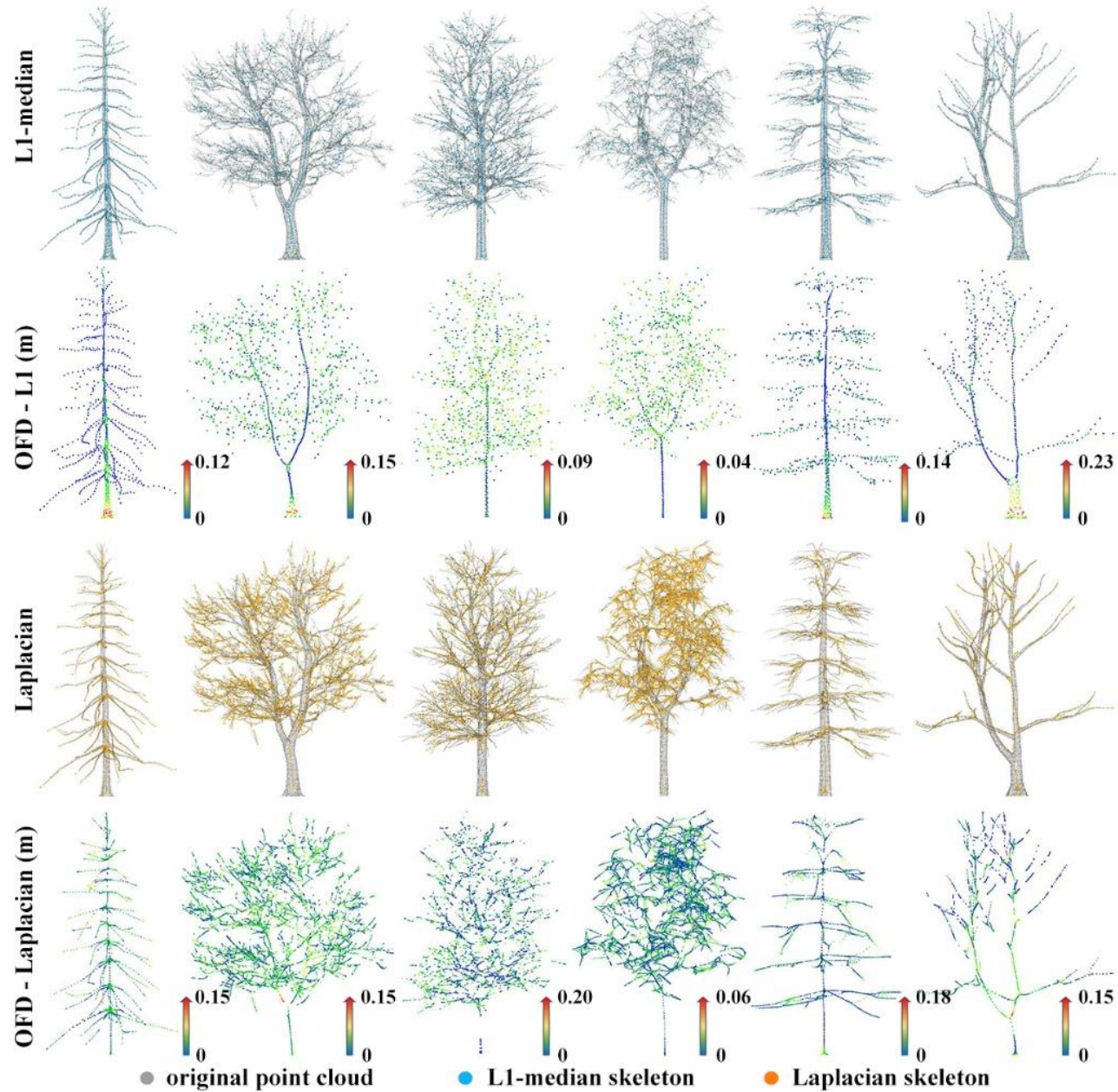

Figure 7 The skeleton extraction results by using the L1-median and Laplacian contraction methods.

The skeleton integrity and extraction accuracy obtained using the Laplacian contraction method were significantly lower than those obtained using the TreeSke and L1-median methods. Based on a visual comparison, we found that the skeleton connectivity of tree forks exacted by the Laplacian contraction method was better than that extracted by the other two tested methods (Figure 7). However, as shown in Table

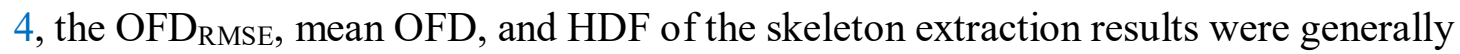
higher for the Laplacian contraction method than for the other two tested methods. Using the Laplacian method could not obtain the median-axis skeleton from woody organs, which caused local distortion and evident missing parts, especially for the trunks. Therefore, we conclude that the Laplacian contraction method is not suitable for extracting skeletons from our tested data. 
The TreeSke-extracted skeleton could present detailed and nearly integral geometrical features of lateral branches and twigs (with $\mathrm{OFD}_{\mathrm{RMSE}}<0.06 \mathrm{~m}$, mean OFD $<0.04 \mathrm{~m}$, and HDF $<0.11 \mathrm{~m}$ ). Thus, the extraction results are more suitable for presenting the structural and phenotypic features of woody materials, such as the surface inclination angle, length, and density of branches. More importantly, there is no need to set any empirical parameters during the extraction. The pointwise spherical searching radius and contraction weight were heuristically set by two local geometrical metrics (LDM and SDM) and the distance between adjacent points. All thresholds were set according to the statistical results of sorted pointwise attributes without empirically setting parameters. To extract the skeleton from data with a variable point density, such as the CA data, we avoided setting the density-related parameters during the coarse skeleton contraction (Mei, Zhang et al. 2016). It should be noted that the geometric information of treetops was always incompletely captured in the field-collected point cloud due to occlusion effects by the nearby foliage and overlapping branches within the forest canopy. The sensor and environmental factors also introduced noise and outliers to the point cloud data (Hosoi and Omasa 2006; Wilkes, Lau et al. 2017). Although the TreeSke method can robustly extract skeletons from a point cloud with noise, outliers, and a small number of missing parts, this method nevertheless has limitations, which will be discussed in the following section.

Table 4 The skeleton extraction accuracy of L1-median and Laplacian contraction methods. In this table, HDF means the Hausdorff distance between the extracted and reference skeletons from each tree model. OFD means the offset distance between each skeleton point to its nearest vertex in the reference skeleton. The $\mathrm{OFD}_{\text {RuSE }}$ is the root mean square error among the pointwise OFD values.

\begin{tabular}{ccccccc}
\hline Data & \multicolumn{3}{c}{ L1-median } & \multicolumn{4}{c}{ Laplacian contraction } \\
\cline { 2 - 7 } & $\begin{array}{c}\text { OFD } \\
(\mathrm{m})\end{array}$ & $\begin{array}{c}\text { Mean OFD } \\
(\mathrm{m})\end{array}$ & $\begin{array}{c}\text { HDF } \\
(\mathrm{m})\end{array}$ & $\begin{array}{c}\text { OFD RUSE } \\
(\mathrm{m})\end{array}$ & $\begin{array}{c}\text { Mean 0FD } \\
(\mathrm{m})\end{array}$ & $\begin{array}{c}\text { HDF } \\
(\mathrm{m})\end{array}$ \\
\hline $\mathrm{AC}$ & 0.08 & 0.04 & 0.14 & 0.11 & 0.09 & 0.13 \\
$\mathrm{GA}$ & 0.12 & 0.10 & 0.17 & 0.11 & 0.10 & 0.22 \\
$\mathrm{SW}$ & 0.11 & 0.09 & 0.14 & 0.12 & 0.09 & 0.14 \\
OR & 0.04 & 0.03 & 0.08 & 0.04 & 0.04 & 0.05 \\
$\mathrm{HS}$ & 0.08 & 0.06 & 0.12 & 0.10 & 0.08 & 0.14 \\
$\mathrm{LP}$ & 0.10 & 0.07 & 0.09 & 0.17 & 0.10 & 0.14 \\
\hline
\end{tabular}




\section{Limitations and robustness}

The TreeSke-extracted skeleton from the frustum-like root and junction parts of woody materials hardly presented a smooth linear distribution, and the same was observed for the L1-median extraction results. We found that the local geometrical features of point sets from these parts were irregular and the extracted skeletons might show a lumpy distribution and deviate from the median-axis of woody organs. Due to the presence of the interlacing branches and twigs, the local point sets searched by $\mathrm{R}_{\mathrm{op}}$ radius (calculated by Eq. 1) for the target point from one woody organ might contain points from its nearby woody organs. This inevitably resulted in distorted skeleton extraction results, especially at the treetop and tree fork parts. Thus, we relocated the skeleton to the median-axis of woody organs according to the ellipse-fitting algorithm (Halir and Flusser 1998). However, the re-centering method is invalid for point clouds from trunks with large numbers of missing parts.

To verify the robustness of our proposed method, we performed a test by extracting a skeleton from the data with random and Gaussian noise, outliers, and missing parts. The results are shown in Figure 5. We found that the noise and outliers had limited efforts on the skeleton extraction results. This is due to the fact that the pointwise contraction weights were set according to two local geometrical metrics (LDM and SDM) rather than the density- or distance-based weights. Although some outliers remained in the thinned skeleton, they would be removed during the skeleton optimization and re-centering steps. However, the missing parts caused obvious skeleton deformation with the pointwise HDF values higher than $0.04 \mathrm{~m}$. The skeleton deformation was more severe for the incomplete point cloud data from trunks. As the TreeSke method uses the LDM and SDM to contract and optimize the point-based skeleton, the missing parts cannot provide information for calculating these two contraction weights. It was infeasible to repair the missing skeletons using the nearby point distribution features as we could not detect the suitable local point sets which showed similar structural features to these missing parts.

Researchers have claimed that some graph-based extraction algorithms are able to 
repair skeletons from missing parts (Tag1iasacchi, Zhang et al. 2009; Mei, Zhang et a1. 2016; Wu, Wen et a1. 2019). However, this is an ill-posed way to construct a graph for a discrete tree point cloud with complex structural features of woody materials. Therefore, these methods are not suitable for our study. Single-station laser scanning or improper multi-station scanning will inevitably cause missing data near the boundary of the scanning range during the data collection. Thus, before skeleton extraction, it is essential to check the structural integrity of the tree point cloud. We think that users should not extract skeletons from single-scan data. Even if they use multi-station data, it is necessary to ensure that there are no large missing parts from trunks; otherwise, skeleton extraction algorithms based on local point distributions would be ineffective, such as the L1-median method and our proposed method.

\section{Applications and Outlook}

To extract the local surface inclination angle of woody materials for modeling the canopy interception process, we designed the TreeSke method to extract skeletons from tree point cloud data. Although the SNV-based method can also be used to evaluate the inclination angle of woody materials and foliage (Ma, Zheng et al. 2017; Liu, Skidmore et a1. 2019), assessing the pointwise SNV requires high-quality point cloud data that are free of noise and missing parts (Bucksch, Lindenbergh et al. 2010; Tagliasacchi, Delame et al. 2016). However, in-situ collected tree point cloud data often contain noise and missing parts caused by the effects of laser scattering or occlusion on the laser transmission path. Additionally, for points from lateral branches with complex structural features, their SNV-based inclination angles might not support the accurate assessment of inclination angle as the extracted local point set might contain points from nearby woody organs. Using the skeletons, we could easily calculate the local inclination angle for lateral branches and twigs by Eq. (16). However, it was difficult to obtain the accurate surface inclination angle of trunks and thick branches using only the skeleton-based method (Figure 6). On the one hand, the skeleton points intersected at the tree forks, thus the local inclination angle assessed by these points cannot show 
617 the surface inclination angles of woody organs. On the other hand, redundant skeleton 618 points at the tree forks and roots also introduced errors in the inclination angle assessment. Setting a simple decision tree based on pointwise LDM values might be beneficial for precisely calculating the surface inclination angle of woody organs. After several tests, for points with LDM values greater than or equal to 0.15 , we used the skeleton-based method to calculate their surface inclination angles as the test points were always from slim branches and twigs. For other points with LDM values lower than 0.15 , we used the SNV-based method to assess their surface inclination angles as they were always from stems and thick branches. By using the above method, as shown in Figure 8, the evaluation accuracy of surface inclination angle could be significantly improved compared to using a single method alone.

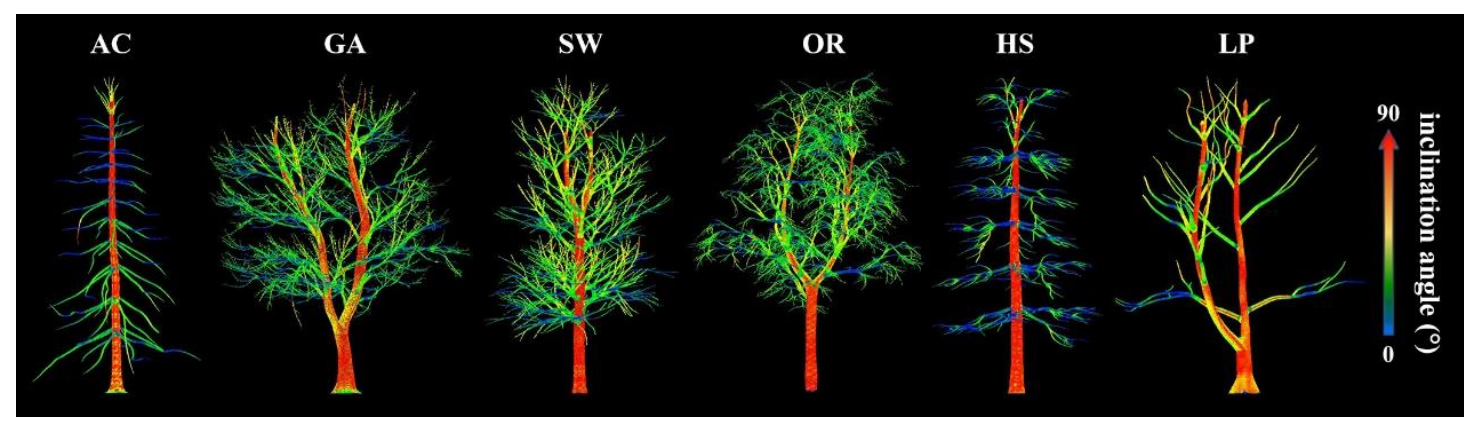

Figure 8 The pointwise surface inclination angle assessed by the optimized method combining the SNV- and skeleton-based methods for six simulated tree point cloud datasets.

Base on the TreeSke-extracted skeleton, users can evaluate the directional projection area and density-related features of woody materials at the plot level. These two types of canopy geometrical features are essential for simulating the shadow area of solar direct radiation and the probable transmission path of stemflow (Zirlewagen and von Wilpert 2001; Ma, Zheng et al. 2017; Zhu, Skidmore et al. 2018). The skeleton distribution features are also important prerequisites for evaluating the health status of epiphyte, the spatial distribution probability of drip water, and air turbulence within forest canopy (Xiao, McPherson et al. 2000; Zimmermann, Wilcke et al. 2007;

640 Germer, Werther et al. 2010; Carlyle-Moses and Gash 2011). Comparing the skeletons 641 extracted from multi-temporal TLS datasets, we can also assess the variation in timber 642 volume and the geometrical characteristics of trunk cross-sections to monitor the health 
of forests (Bienert, Hess et a1. 2014).

The TreeSke method can directly extract the skeleton of woody materials from a discrete point cloud data. Before using this method, it is necessary to identify the woody and foliage points from the whole tree point cloud data, which can be separated by many novel machine learning algorithms (Zhu, Skidmore et al. 2018; Jin, Su et al. 2019; Wang 2020). With the development and universal application of multi-spectral TLS in forest ecological studies, we expect that the separation accuracy of woody and foliage points will be improved, which will allow the more detailed extraction of the skeletons of woody materials in dense canopy. The extracted skeletons can support the reconstruction of the missing parts of point cloud data, which is a research hotspot in the computing vision field (Yan, Wintz et al. 2009; Livny, Yan et a1. 2010; Zhang, Bao et al. 2017). In the following research, we plan to combine 3-D deep learning algorithms and TreeSke-extracted skeletons to repair tree point cloud data with missing parts.

\section{Conclusions}

In this study, we designed the TreeSke method to extract the median-axis skeletons from the discrete point cloud data of woody materials. This method firstly contracted the coarse skeleton by pointwise heuristically searching the local point set and using the median-weighted method to relocate each point. Then, we iteratively thinned the coarse skeleton by the range-weighted average method, where the range means the shortest Euclidean distance between the thinning skeleton and original point cloud data. After filtering noise and point re-centering processes, we could obtain the skeletons of each woody material. Comparing with other tested methods, the high merit of TreeSke is that the extracted median-axis skeletons maintain the structural lossless features of

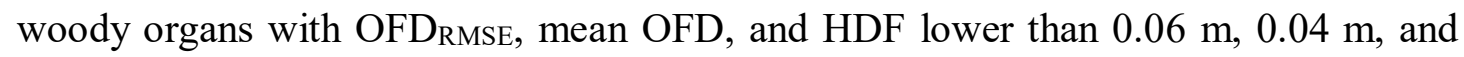
$0.11 \mathrm{~m}$, respectively. Moreover, the TreeSke method could extract skeletons from the field-collected data at the plot level. We also verified its robustness to low-quality data 
670 with noise and outliers. However, the missing parts in point cloud data would reduce

671 the skeleton integrity and cause deformation issue. The TreeSke-extracted skeleton 672 shows the potential to analyze the phenotypic features of woody materials within the 673 forest canopy, and thus to support for simulating the canopy interception allocation and understory distribution of solar radiation in our following researches.

\section{Abbreviations}

676 RMSE: Root Mean Square Error;

677 TLS: Terrestrial laser scanner;

678 OFD: Offset distance between the extracted and reference skeleton of scan targets;

$679 \mathrm{OFD}_{\text {Ruse }}$ : the root mean square error among the point-to-point offset distance values;

680 SNV: Surface normal vector of point cloud data;

681 DBSCAN: Density-based spatial clustering of applications with noise algorithm;

682 CNN: Convolutional Neural Network;

683 KDtree: K dimensional tree searching index based on the pointwise 3-D coordinates;

684 MST: Minimum Spanning Tree;

685 LDM: Linear degree measures of local point set distribution;

686 SDM: Spherical degree measures of local point set distribution;

$687 \mathrm{P}_{\mathrm{ve}}$ : The pointwise local point set;

$688 \mathrm{R}_{\mathrm{op}}$ : The pointwise searching radius of local point set for each point in the whole 689 point cloud data;

$690 \quad \mathrm{D}_{\mathrm{co}}$ : The shortest pointwise Euclidean distance between the extracted coarse skeleton 691 and original point cloud data;

$692 \mathrm{C}_{\text {cov }}$ : The covariance matrix of $\mathrm{n}(\mathrm{n} \geqslant 3)$ coordinates;

693 SUM $_{d}$ : The sum of the Euclidean distance from a point to its $k$ ( $k=9$ in this study)

694 neighboring points;

695 HDF: The Hausdorff distance between the extracted and reference skeletons for each

696 simulated tree model;

697 MP: The Masson's Pines (Pinus massoniana) plot;

698 CA: The Chinese Aspens (Populus adenopoda) plot;

699 AC: The Alaska Cedar (Chamaecyparis lawsoniana) model;

700 GA: The Green Ash (Fraxinus pennsylvanica Marsh) mode1;

701 SW: The Sourwood (Oxydendrum arboreum) model;

702 HS: The Huangshan Pine (Pinus taiwanensis Hayata) mode1;

703 LP: The London Plane (Platanus acerifolia) model;

704 OR: The Orange (Glycosmis cochinchinensis) tree model;

705 IA: The inclination angle 
We really thank the Red Soil Ecological Experimental Station of the Institute of Soil Science, Chinese Academy of Sciences for supporting us for collecting the point cloud data of natural forests. We also thank the developers of the Starlab, Laplacian contraction, and L1-median software.

\section{Funding}

712 This research was funded by the State Key Laboratory of Soil and Sustainable 713 Agriculture Research Fund (Y812000002), National Science Foundation of China (NSFC)

714 (NSFC award \#41771374 and NSFC award \#41671343), and the program B for Outstanding 715 PhD candidate of Nanjing University.

\section{Author Contributions}

717 Conceptualization, B.W. and G. Z. ; methodology and software, B.W. ; data collection and process, B.W. and Y.C. ; validation, Y.C. ; writing-original draft preparation, B.W. ; writing-review and editing, G.Z. ; project administration, G.Z. ; funding 720 acquisition, G.Z. All authors have read and agreed to the published version of the manuscript.

\section{Ethics approval and consent to participate}

Not applicable.

\section{Consent for publication}

Not applicable.

\section{Availability of data and materials}

727 The datasets used and/or analysed during the current study are available from the corresponding author on reasonable request.

\section{Competing interests}


732

1 State Key Laboratory of Soil and Sustainable Agriculture, Institute of Soil Science, Chinese

Academy of Sciences, Nanjing 210008, China;

2 International Institute for Earth System Science, Nanjing University, Nanjing 210023, China;

3 University of Chinese Academy of Sciences, Beijing 100049, China

Received: 15 September, 2020

Accepted:

\section{References}

Ashcroft MB, JR Gollan, D Ramp, D Kriticos (2014) Creating vegetation density profiles for a diverse range of ecological habitats using terrestrial laser scanning. Methods in Ecology and Evolution 5:263-272. doi: 10.1111/2041-210x. 12157

Au OK-C, C-L Tai, H-K Chu, D Cohen-0r, T-Y Lee (2008) Skeleton extraction by mesh contraction. ACM transactions on graphics (TOG) 27:1-10

Bienert A, C Hess, HG Maas, G von Oheimb (2014) A voxel-based technique to estimate the volume of trees from terrestrial laser scanner data. ISPRS - International Archives of the Photogrammetry, Remote Sensing and Spatial Information Sciences XL-5:101-106. doi: 10. 5194/isprsarchives-XL-5-101-2014

Bremer M, M Rutzinger, V Wichmann (2013) Derivation of tree skeletons and error assessment using LiDAR point cloud data of varying quality. ISPRS journal of photogrammetry and remote sensing 80:39-50

Bucksch A, R Lindenbergh (2008) CAMPINO - A skeletonization method for point cloud processing. ISPRS Journal of Photogrammetry and Remote Sensing 63:115-127. doi: 10. 1016/j. isprsjprs. 2007. 10. 004

Bucksch A, R Lindenbergh, M Menenti (2010) Ske1Tre. The Visual Computer 26:1283-1300. doi : 10. 1007/s00371-010-0520-4

Cao J, A Tagliasacchi, M 01son, H Zhang, Z Su (2010) Point Cloud Skeletons via Laplacian Based Contraction. 2010 Shape Modeling International Conference, pp. $187-197$

Carlyle-Moses DE, JHC Gash (2011) Rainfall Interception Loss by Forest Canopies. Forest Hydrology and Biogeochemistry, pp. 407-423

De Schrijver A, G Geudens, L Augusto, J Staelens, J Mertens, K Wuyts, L Gielis, K Verheyen (2007) The effect of forest type on throughfall deposition and seepage flux: a review. Oecologia 153:663-674. doi: 10.1007/s00442-007-07761

Digumarti ST, J Nieto, C Cadena, R Siegwart, P Beardsley (2018) Automatic Segmentation of Tree Structure From Point Cloud Data. IEEE Robotics and Automation Letters 3:3043-3050. doi: 10. 1109/1ra. 2018. 2849499

Disney M (2018) Terrestrial LiDAR : a three-dimensional revolution in how we look 
at trees. New Phytologist 222:1736-1741. doi: 10.1111/nph. 15517

Ferrara R, SGP Virdis, A Ventura, T Ghisu, P Duce, G Pellizzaro (2018) An automated approach for wood-leaf separation from terrestrial LIDAR point clouds using the density based clustering algorithm DBSCAN. Agricultural and Forest Meteorology 262:434-444. doi: 10.1016/j. agrformet. 2018. 04. 008

Gash JHC, CR Lloyd, G Lachaud (1995) Estimating Sparse Forest Rainfall Interception with an Analytical Model. Journal of Hydrology 170:79-86. doi: Doi 10. 1016/0022-1694 (95) 02697-N

Germer S, L Werther, H Elsenbeer (2010) Have we underestimated stemflow? Lessons from an open tropical rainforest. Journal of Hydrology 395:169-179. doi: 10. 1016/j. jhydro1. 2010. 10. 022

Halr R, J Flusser (1998) Numerically stable direct least squares fitting of ellipses. Proc 6th International Conference in Central Europe on Computer Graphics and Visualization WSCG. Citeseer, pp. 125-132

Hanchi A, M Rapp (1997) Stemflow determination in forest stands. Forest Ecol Manag $97: 231-235$

Hancock S, R Essery, T Reid, J Carle, R Baxter, N Rutter, B Huntley (2014) Characterising forest gap fraction with terrestrial lidar and photography: An examination of relative limitations. Agricultural and Forest Meteorology 189-190:105-114. doi: 10. 1016/j. agrformet. 2014.01. 012

Hosoi F, K Omasa (2006) Voxel-Based 3-D Modeling of Individual Trees for Estimating Leaf Area Density Using High-Resolution Portable Scanning Lidar. IEEE Transactions on Geoscience and Remote Sensing 44:3610-3618. doi: 10. 1109/tgrs. 2006. 881743

Huang H, S Wu, D Cohen-0r, M Gong, H Zhang, G Li, B Chen (2013) L1-medial skeleton of point cloud. ACM Trans Graph 32:65:61-65:68

Jin S, Y Su, S Gao, F Wu, Q Ma, K Xu, T Hu, J Liu, S Pang, H Guan (2019) Separating the structural components of maize for field phenotyping using terrestrial lidar data and deep convolutional neural networks. IEEE Transactions on Geoscience and Remote Sensing 58:2644-2658

Li R, G Bu, P Wang (2017) An Automatic Tree Skeleton Extracting Method Based on Point Cloud of Terrestrial Laser Scanner. International Journal of Optics 2017:111. doi: 10. 1155/2017/5408503

Li Y, Y Su, T Hu, G Xu, Q Guo (2018) Retrieving 2-D Leaf Angle Distributions for Deciduous Trees From Terrestrial Laser Scanner Data. IEEE Transactions on Geoscience and Remote Sensing 56:4945-4955. doi: 10. 1109/tgrs. 2018. 2843382

Li Y, Y Su, X Zhao, M Yang, T Hu, J Zhang, J Liu, M Liu, Q Guo (2020) Retrieval of tree branch architecture attributes from terrestrial laser scan data using a Laplacian algorithm. Agricultural and Forest Meteorology 284. doi: 10. 1016/j. agrformet. 2019. 107874

Lin Y, J Liu, J Zhou (2020) A novel tree-structured point cloud dataset for skeletonization algorithm evaluation. arXiv preprint arXiv:200102823

Liu J, AK Skidmore, T Wang, X Zhu, J Premier, M Heurich, B Beudert, S Jones (2019) Variation of leaf angle distribution quantified by terrestrial LiDAR in 
natural European beech forest. ISPRS Journal of Photogrammetry and Remote Sensing 148:208-220. doi: 10.1016/j. isprsjprs. 2019.01.005

Liu Y, X Mu, H Wang, G Yan, G Henebry (2012) A novel method for extracting green fractional vegetation cover from digital images. Journal of Vegetation Science 23:406-418. doi: 10.1111/j. 1654-1103. 2011.01373. x

Livny Y, F Yan, M 01son, B Chen, H Zhang, J E1-Sana (2010) Automatic reconstruction of tree skeletal structures from point clouds. ACM SIGGRAPH Asia 2010 papers on - SIGGRAPH ASIA' 10

Ma L, G Zheng, JUH Eitel, TS Magney, LM Moskal (2017) Retrieving forest canopy extinction coefficient from terrestrial and airborne lidar. Agricultural and Forest Meteorology 236:1-21. doi: 10.1016/j. agrformet. 2017.01. 004

Maurer KD, BS Hardiman, CS Voge1, G Bohrer (2013) Canopy-structure effects on surface roughness parameters: Observations in a Great Lakes mixed-deciduous forest. Agricultural and forest meteorology 177:24-34

Mei J, L Zhang, S Wu, Z Wang, L Zhang (2016) 3D tree modeling from incomplete point clouds via optimization and L1-MST. International Journal of Geographical Information Science 31:999-1021. doi: 10. 1080/13658816. 2016. 1264075

Mitchel1 PJ, PNJ Lane, RG Benyon (2012) Capturing within catchment variation in evapotranspiration from montane forests using LiDAR canopy profiles with measured and modelled fluxes of water. Ecohydrology 5:708-720. doi: 10. $1002 /$ eco. 255

Ozbay E, A Cinar, Z Guler (2018) A hybrid method for skeleton extraction on Kinect sensor data: Combination of L 1 -Median and Laplacian shrinking algorithms. Measurement 125:535-544. doi: 10.1016/j. measurement. 2018.05. 029

Pereira FL, JHC Gash, JS David, F Valente (2009) Evaporation of intercepted rainfall from isolated evergreen oak trees: Do the crowns behave as wet bulbs? Agricultural and Forest Meteorology 149:667-679. doi: 10. 1016/j. agrformet. 2008. 10. 013

Qin H, J Han, N Li, H Huang, B Chen (2019) Mass-driven topology-aware curve skeleton extraction from incomplete point clouds. IEEE Transactions on Visualization and Computer Graphics

Ryu Y, 0 Sonnentag, T Nilson, R Vargas, H Kobayashi, R Wenk, DD Baldocchi (2010) How to quantify tree leaf area index in an open savanna ecosystem: A multiinstrument and multi-model approach. Agricultural and Forest Meteorology 150:63-76. doi: 10.1016/j. agrformet. 2009. 08.007

Saarinen N, V Kankare, M Vastaranta, V Luoma, J Pyörälä, T Tanhuanpää, X Liang, H Kaartinen, A Kukko, A Jaakkola, X Yu, M Holopainen, J Hyyppä (2017) Feasibility of Terrestrial laser scanning for collecting stem volume information from single trees. ISPRS Journal of Photogrammetry and Remote Sensing 123:140-158. doi: 10.1016/j. isprsjprs. 2016.11. 012

Schumacher J, JR Christiansen (2015) Forest canopy water fluxes can be estimated using canopy structure metrics derived from airborne light detection and ranging (LiDAR). Agricultural and Forest Meteorology 203:131-141

Song C, Z Pang, X Jing, C Xiao (2016) Distance field guided L1-median skeleton 
extraction. The Visual Computer 34:243-255. doi: 10. 1007/s00371-016-1331-z

Sun J, X Yu, H Wang, G Jia, Y Zhao, Z Tu, W Deng, J Jia, J Chen (2018) Effects of forest structure on hydrological processes in China. Journal of Hydrology 561:187-199. doi: 10. 1016/j. jhydro1. 2018. 04. 003

Tagliasacchi A, I Alhashim, M 01son, H Zhang (2012) Mean curvature skeletons. Computer Graphics Forum. Wiley Online Library, pp. 1735-1744

Tagliasacchi A, T Delame, M Spagnuolo, N Amenta, A Telea (2016) 3D skeletons: A state - of - the - art report. Computer Graphics Forum. Wiley Online Library, pp. $573-597$

Tagliasacchi A, H Zhang, D Cohen-0r (2009) Curve skeleton extraction from incomplete point cloud. ACM SIGGRAPH 2009 papers, pp. 1-9

Taha AA, A Hanbury (2015) An efficient algorithm for calculating the exact Hausdorff distance. IEEE Trans Pattern Anal Mach Inte11 37:2153-2163. doi: 10. 1109/TPAMI. 2015. 2408351

Vicari MB, J Pisek, M Disney (2019) New estimates of leaf angle distribution from terrestrial LiDAR: Comparison with measured and modelled estimates from nine broadleaf tree species. Agricultural and Forest Meteorology 264:322-333. doi: 10. 1016/j. agrformet. 2018. 10. 021

Wang D (2020) Unsupervised semantic and instance segmentation of forest point clouds. ISPRS Journal of Photogrammetry and Remote Sensing 165:86-97

Wei S, T Yin, MA Dissegna, AJ Whittle, GLF Ow, MLM Yusof, N Lauret, J-P GastelluEtchegorry (2020) An assessment study of three indirect methods for estimating leaf area density and leaf area index of individual trees. Agricultural and Forest Meteorology 292-293. doi: 10. 1016/j. agrformet. 2020. 108101

Wilkes P, A Lau, M Disney, K Calders, A Burt, J Gonzalez de Tanago, H Bartholomeus, B Brede, M Herold (2017) Data acquisition considerations for Terrestrial Laser Scanning of forest plots. Remote Sensing of Environment 196:140-153. doi: 10. 1016/j. rse. 2017.04. 030

Wu B, G Zheng, Y Chen (2020) An Improved Convolution Neural Network-Based Model for Classifying Foliage and Woody Components from Terrestrial Laser Scanning Data. Remote Sensing 12:1010

Wu S, W Wen, B Xiao, X Guo, J Du, C Wang, Y Wang (2019) An Accurate Skeleton Extraction Approach From 3D Point Clouds of Maize Plants. Front Plant Sci 10:248. doi: 10.3389/fpls. 2019. 00248

Xiao Q, EG McPherson, SL Ustin, ME Grismer (2000) A new approach to modeling tree rainfall interception. Journal of Geophysical Research: Atmospheres $105: 29173-29188$

Yan D-M, J Wintz, B Mourrain, W Wang, F Boudon, C Godin (2009) Efficient and robust reconstruction of botanical branching structure from laser scanned points. 2009 11th IEEE International Conference on Computer-Aided Design and Computer Graphics. IEEE, pp. 572-575

Yan G, R Hu, J Luo, M Weiss, H Jiang, X Mu, D Xie, W Zhang (2019) Review of indirect optical measurements of leaf area index: Recent advances, challenges, and perspectives. Agricultural and Forest Meteorology 265:390-411. doi: 
10. 1016/j. agrformet. 2018. 11. 033

Zhang W, J Qi, P Wan, H Wang, D Xie, X Wang, G Yan (2016) An Easy-to-Use Airborne LiDAR Data Filtering Method Based on Cloth Simulation. Remote Sensing 8. doi: 10. 3390/rs8060501

Zhang X, G Bao, W Meng, M Jaeger, H Li, O Deussen, B Chen (2017) Tree branch leve1 of detail models for forest navigation. Computer Graphics Forum. Wiley Online Library, pp. 402-417

Zhu X, AK Skidmore, R Darvishzadeh, K0 Niemann, J Liu, Y Shi, T Wang (2018) Foliar and woody materials discriminated using terrestrial LiDAR in a mixed natural forest. International Journal of Applied Earth Observation and Geoinformation 64:43-50. doi: 10. 1016/j. jag. 2017.09. 004

Zhu X, AK Skidmore, T Wang, J Liu, R Darvishzadeh, Y Shi, J Premier, M Heurich (2018) Improving leaf area index (LAI) estimation by correcting for clumping and woody effects using terrestrial laser scanning. Agricultural and Forest Meteorology 263:276-286. doi: 10.1016/j. agrformet. 2018.08. 026

Zimmermann A, W Wilcke, H Elsenbeer (2007) Spatial and temporal patterns of throughfall quantity and quality in a tropical montane forest in Ecuador. Journal of Hydrology 343:80-96. doi: 10. 1016/j. jhydro1. 2007.06. 012

Zirlewagen D, K von Wilpert (2001) Modeling water and ion fluxes in a highly structured, mixed-species stand. Forest Ecol Manag 143:27-37. doi: Doi 10. 1016/S0378-1127 (00) 00522-3

Zou J, G Yan, L Zhu, W Zhang (2009) Woody-to-total area ratio determination with a multispectral canopy imager. Tree Physiology 29:1069-1080. doi: 10. 1093/treephys/tpp042 


\section{Figures}
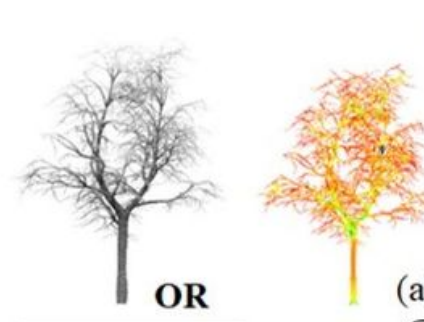

SDM

LDM

ti.

(a)
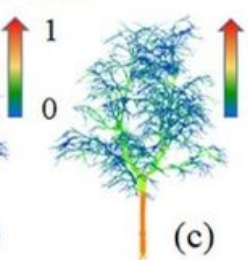

Rop (m)
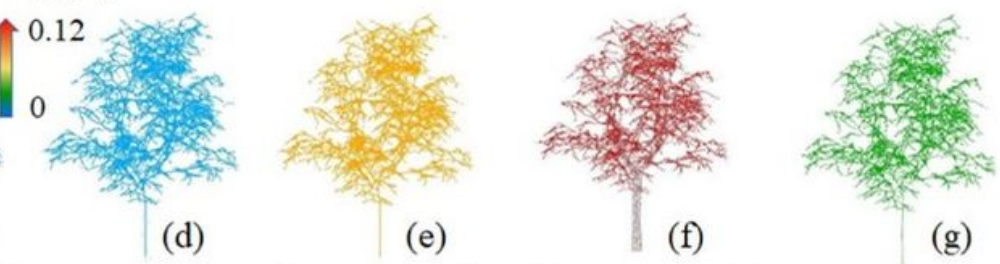

(g)
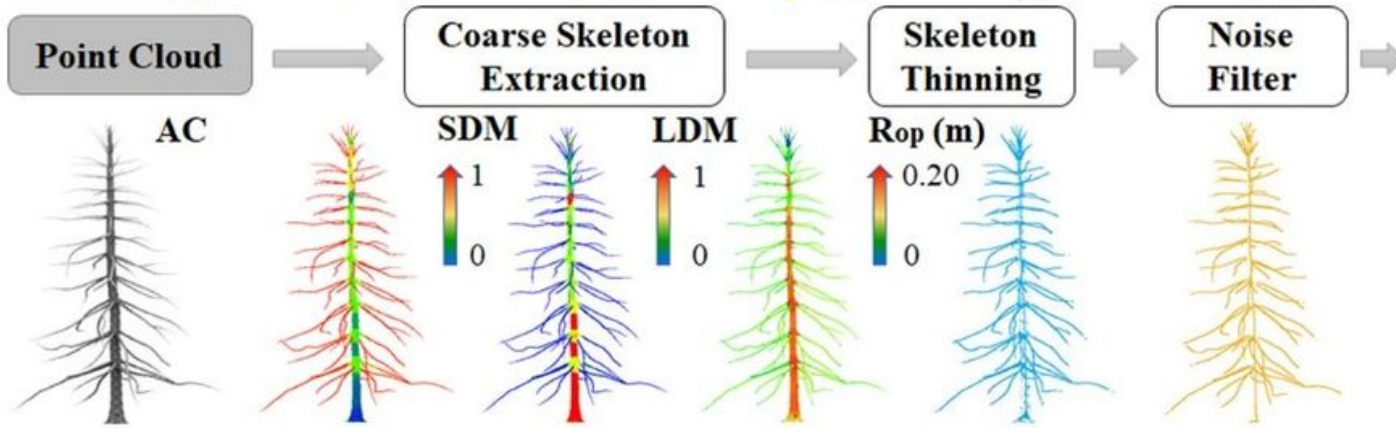

Re-
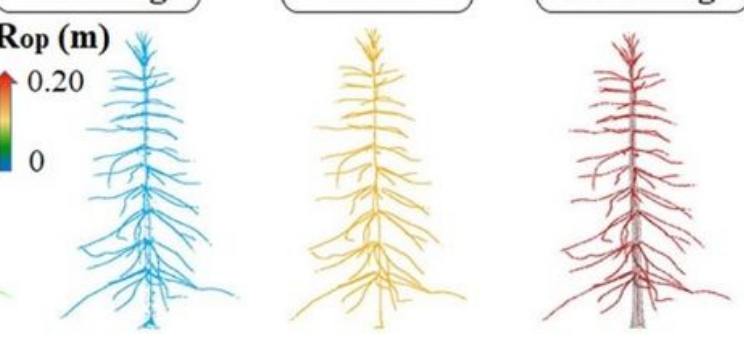

Skeleton

\section{Figure 1}

The flowchart of the TreeSke method for skeleton extraction and the step-by-step extraction results of two tree point cloud (OR and $A C$ ) data. Rop means the pointwise searching radius of local point sets in the whole point cloud data. The LDM and SDM show the linear and spherical degree measures of local point set distribution, respectively. The subfigures (a) to (c) show the evaluation results of pointwise Rop, LDM, and SDM for two tested point cloud data. 


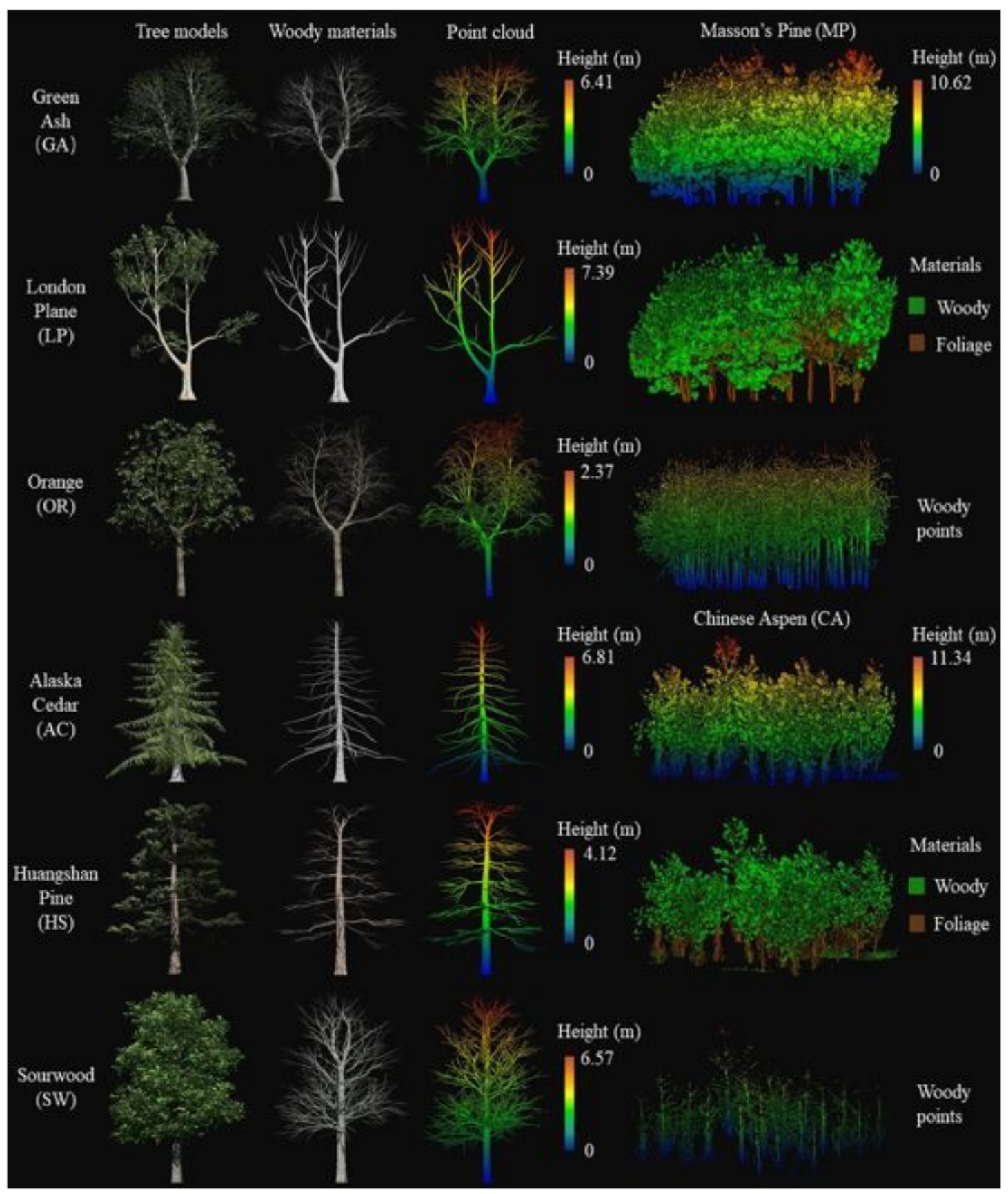

\section{Figure 2}

The point cloud of woody materials converted from six simulated single tree mesh models and those collected from MP and CA plots. 


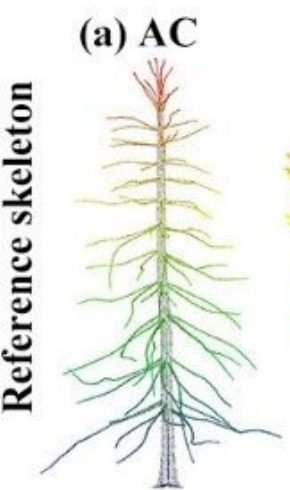

(g)

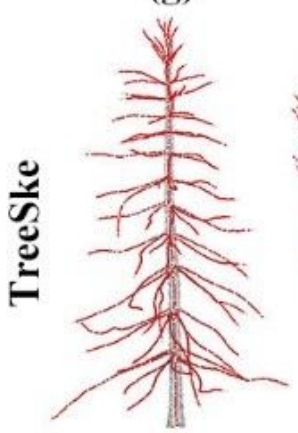

(h)

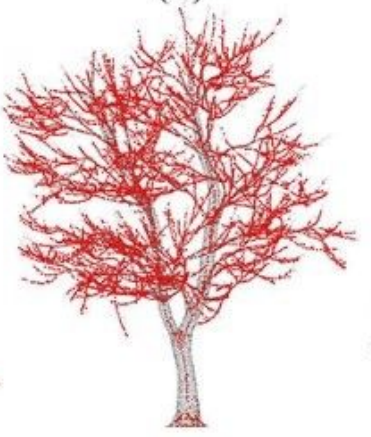

(m) MP plot

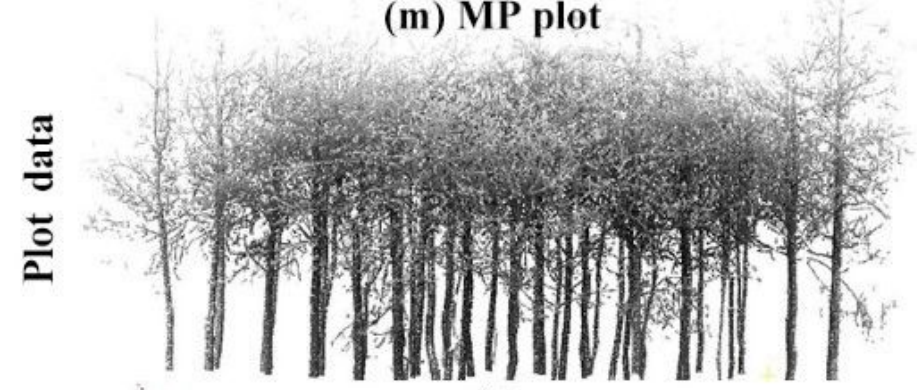

(o)

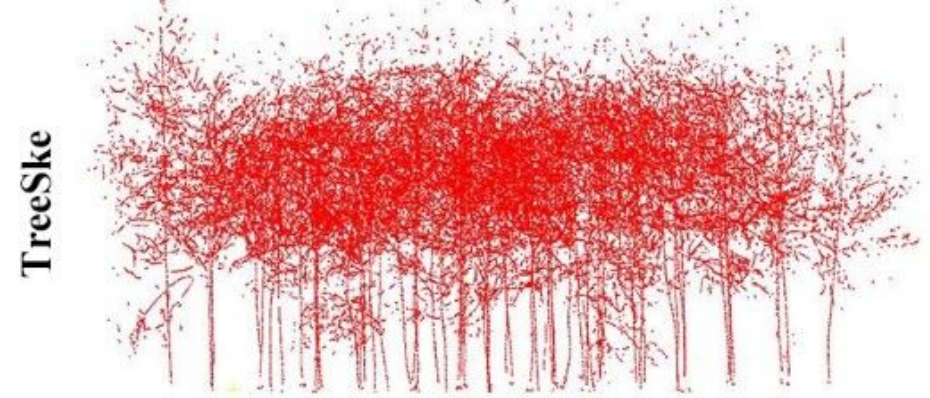

original point cloud (d) $\mathrm{OR}$

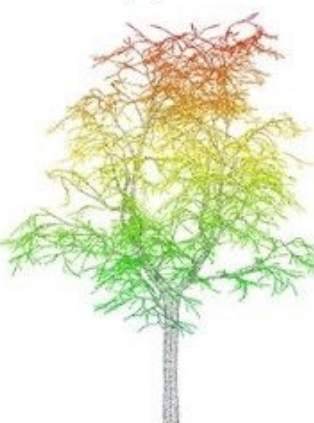

(j)
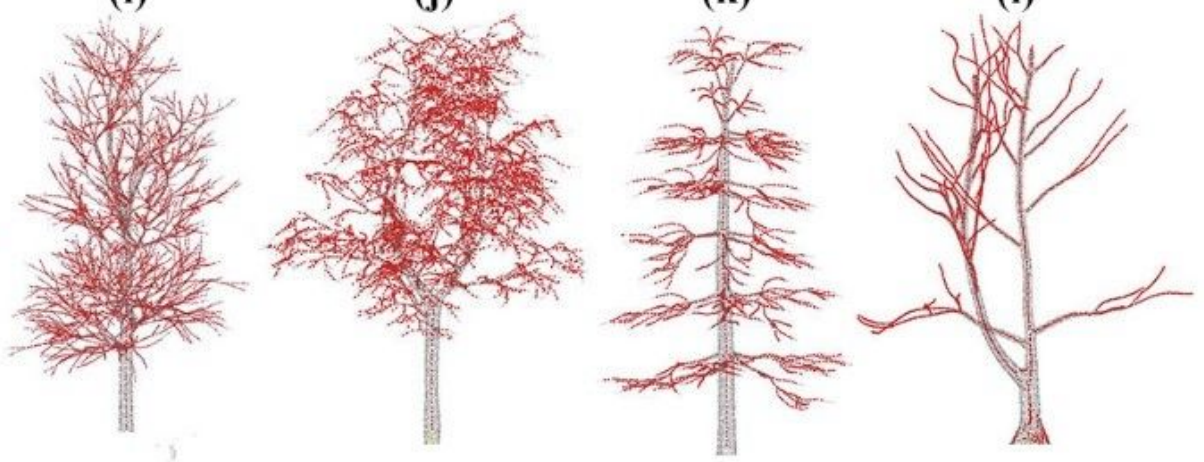

(n) CA plot (k)

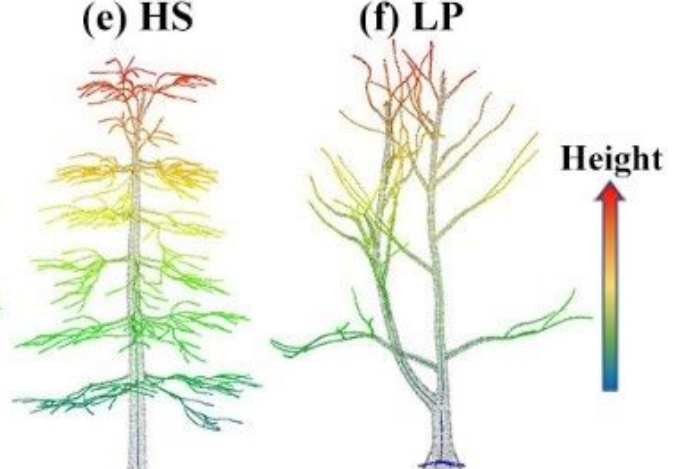

(I)

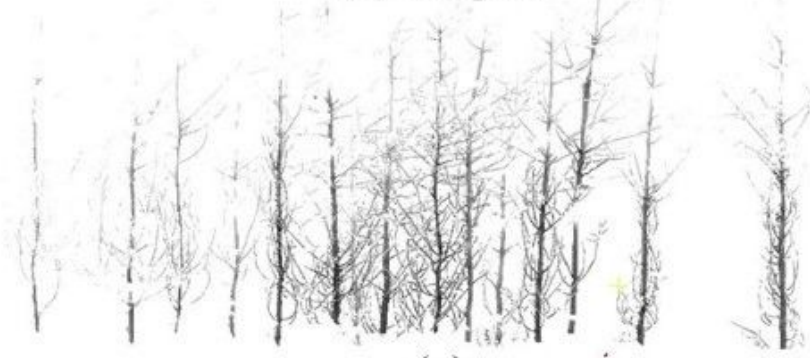

(p)

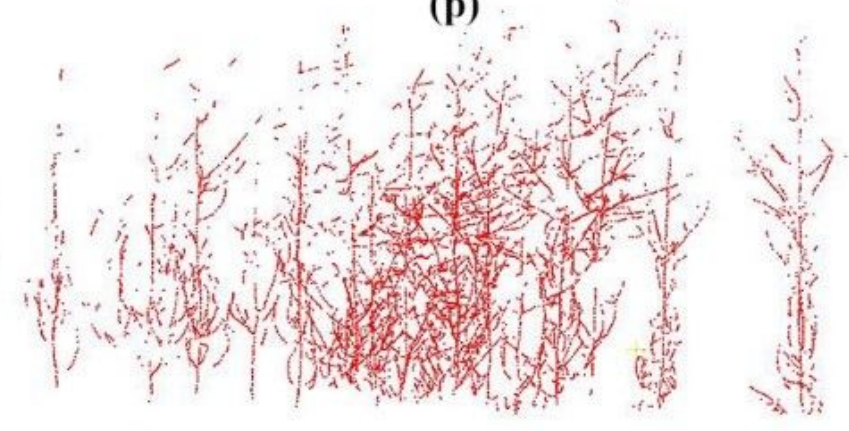

skeleton points

\section{Figure 3}

The TreeSke-extracted results of simulated (single tree level) and field-collected (plot-level) tree point cloud datasets. 
(a) $\mathrm{AC}$

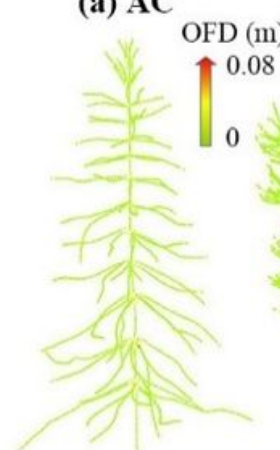

HDF: $0.07 \mathrm{~m}$ RMSE: $0.02 \mathrm{~m}$ Mean OFD: $0.01 \mathrm{~m}$ (b) GA

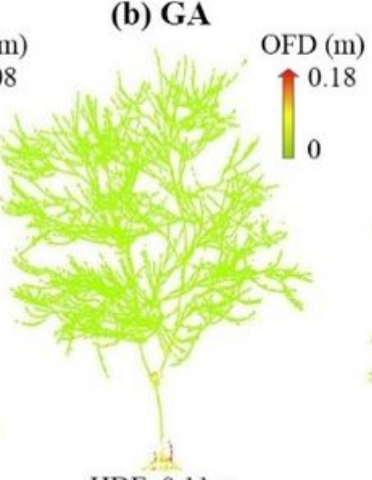

HDF: $0.11 \mathrm{~m}$

RMSE: $0.03 \mathrm{~m}$ Mean OFD: $0.02 \mathrm{~m}$ (c) SW

OFD $(\mathrm{m})$
-0.08

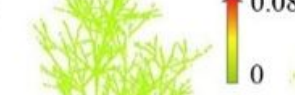

HDF: $0.06 \mathrm{~m}$

RMSE: $0.02 \mathrm{~m}$

Mean OFD: $0.01 \mathrm{~m}$ (d) OR

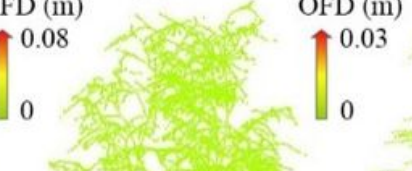

(e) $\mathrm{HS}$

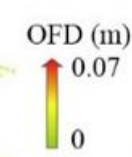

(f) LP

OFD (m) 0.18

\section{Figure 4}

The pointwise OFD of TreeSke-extracted skeleton points. In each inset, the gradient colors show the variation trend of pointwise OFD values, which means the offset distance from each extracted skeleton to its corresponding reference skeleton. RMSE means the RMSE of pointwise OFD values from a single tree's data. 

(a) $\mathrm{AC}$
(b) GA
(c) $\mathrm{SW}$
(d) OR
(e) HS
(f) LP
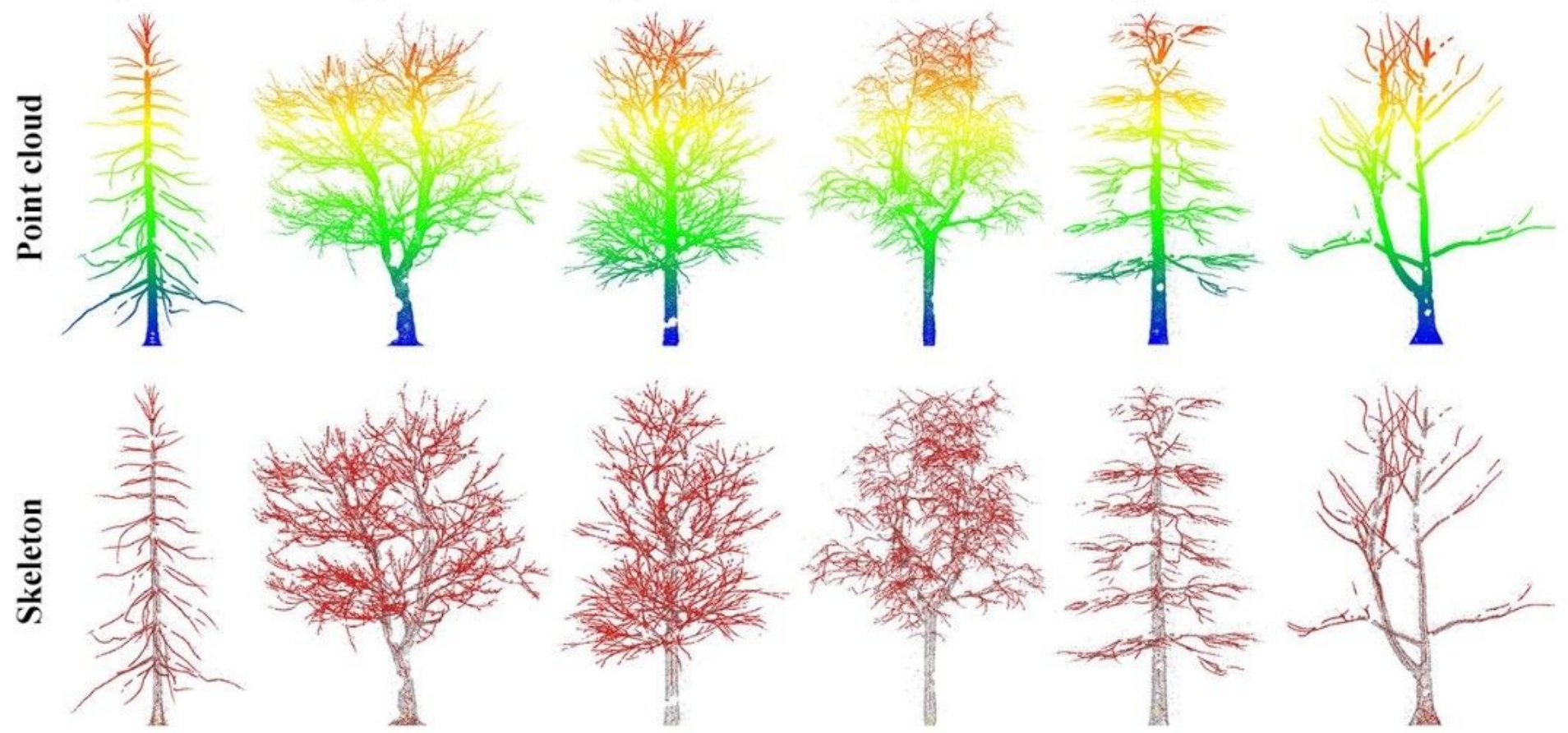

original point cloud

- skeleton point
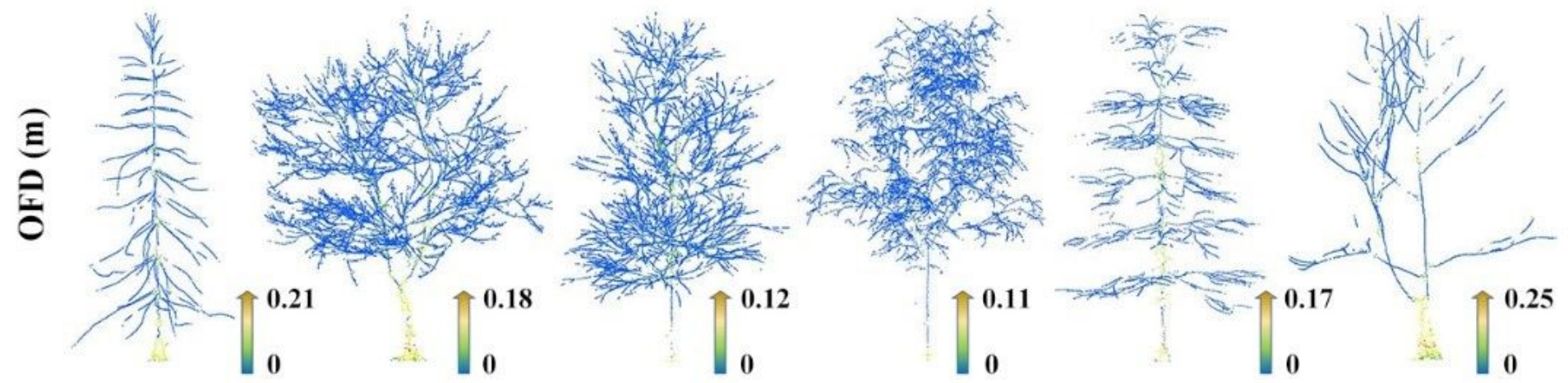

Figure 5

The TreeSke-extracted skeletons and pointwise OFD values of point cloud data with noisy points, outliers, and missing parts. 


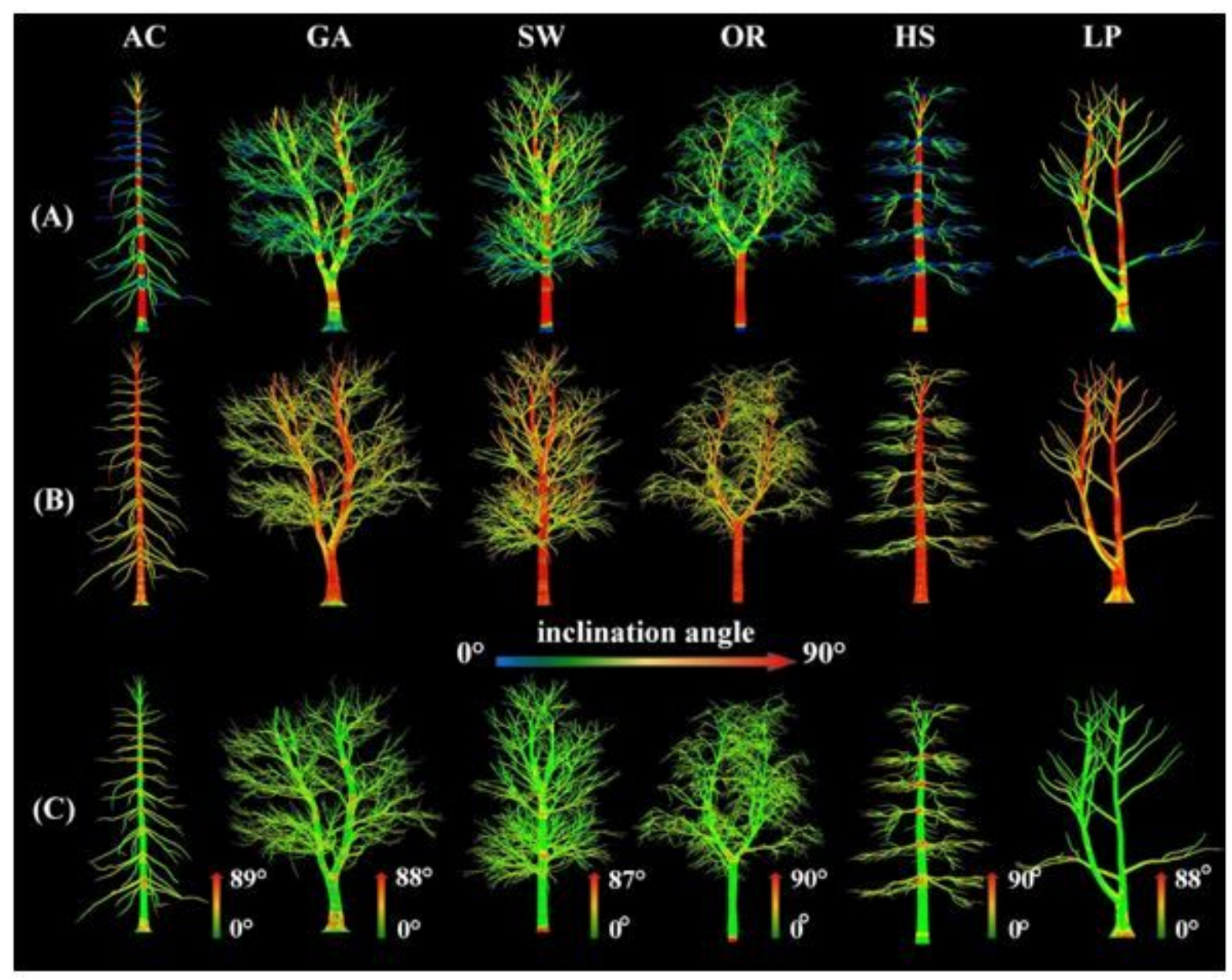

\section{Figure 6}

The insets in row (A) and (B) show the pointwise surface inclination angles calculated by the skeletonand SNV-based methods, respectively. In row (C), these insets show the pointwise absolute difference of surface inclination angle evaluated by two methods. 

(a) $\mathrm{AC}$
(b) GA
(c) $\mathrm{SW}$
(d) OR
(e) HS
(f) LP
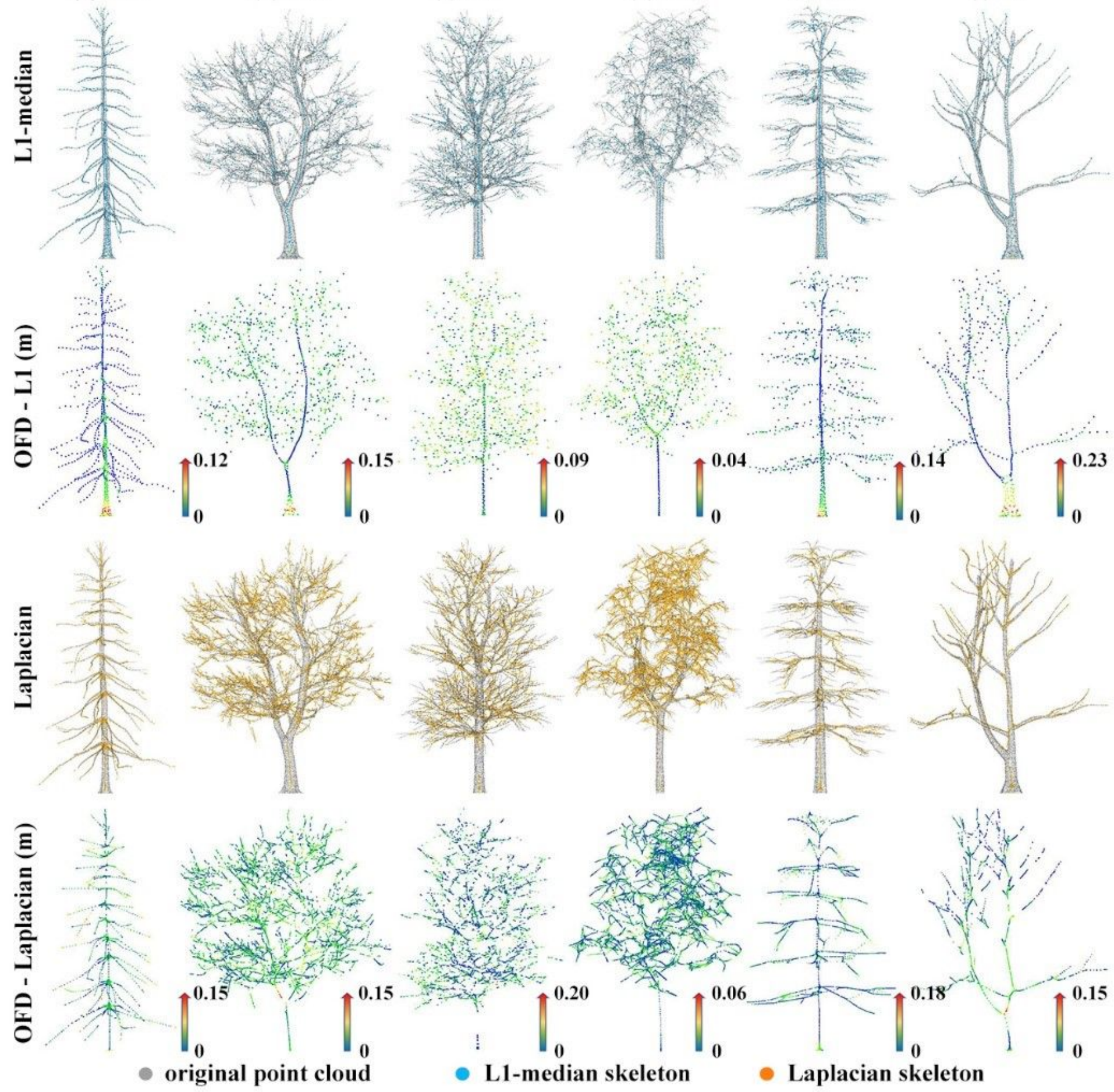

L1-median skeleton

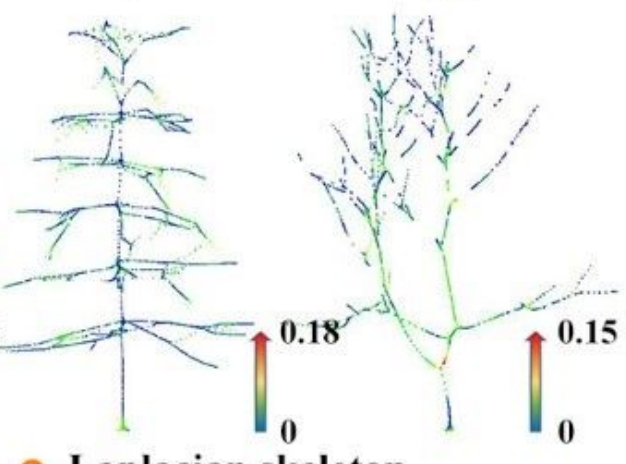

Laplacian skeleton

Figure 7

The skeleton extraction results by using the L1-median and Laplacian contraction methods. 


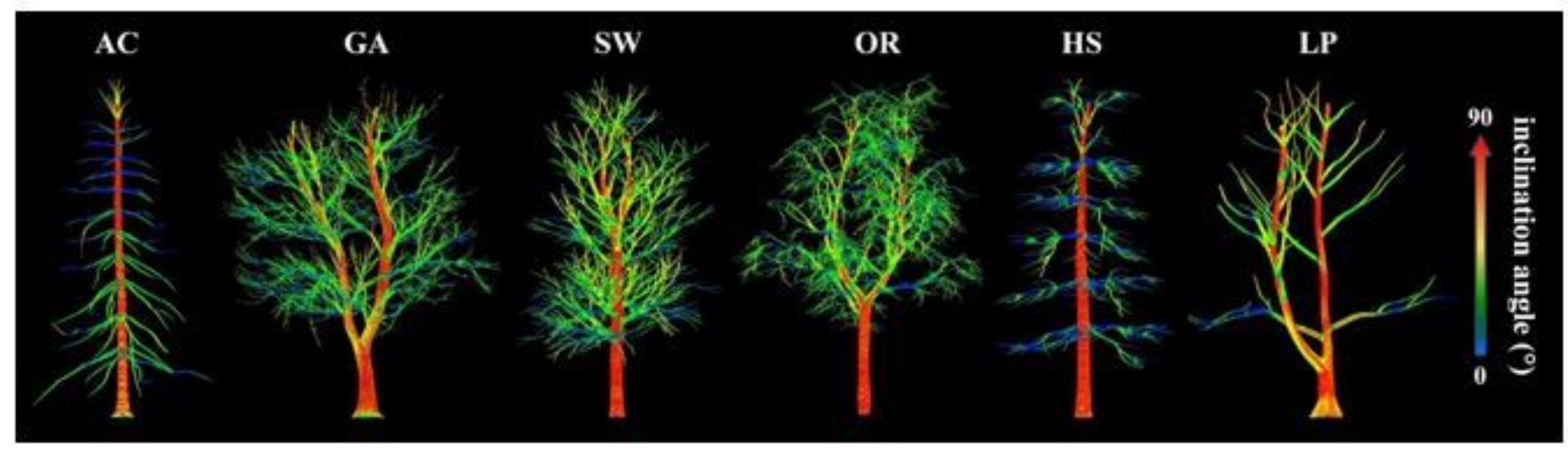

Figure 8

The pointwise surface inclination angle assessed by the optimized method combining the SNV-and skeleton-based methods for six simulated tree point cloud datasets. 